\title{
EXISTENCE FOR AN UNSTEADY FLUID-STRUCTURE INTERACTION PROBLEM
}

\author{
CÉline Grandmont ${ }^{1}$ And Yvon MadaY ${ }^{1,2}$
}

\begin{abstract}
We study the well-posedness of an unsteady fluid-structure interaction problem. We consider a viscous incompressible flow, which is modelled by the Navier-Stokes equations. The structure is a collection of rigid moving bodies. The fluid domain depends on time and is defined by the position of the structure, itself resulting from a stress distribution coming from the fluid. The problem is then nonlinear and the equations we deal with are coupled. We prove its local solvability in time through two fixed point procedures.
\end{abstract}

Mathematics Subject Classification. 76D05, 35Q30, 73K70.

Received: April 9, 1999. Revised: October 7, 1999.

\section{INTRODUCTION}

The problem we study deals with fluid-structure interaction in the case where the fluid domain is time dependent and the structure is a collection of rigid bodies. For related works on this type of problems we refer to $[6-8,15]$ and to [11] for numerical simulations. We consider a $2 \mathrm{D}$ or $3 \mathrm{D}$, viscous, incompressible flow satisfying the Navier-Stokes equations. We denote by $\nu$ the fluid viscosity. We suppose that the fluid fills, at time $t=0$, a smooth domain of $\mathbb{R}^{d}, d=2,3$. In this cavity denoted by $\Omega$, we consider a collection of rigid but moving bodies $\mathcal{B}_{i}, 1 \leq i \leq N$ with density $\rho_{i}$, mass $m_{i}$ and matrix of inertia $J_{i}$. We denote by $\mathcal{B}_{i}(t)$, the position of the $i^{t h}$ body at time $t$. Their motion is thus described by three or six degrees of freedom: translations and rotations. If we denote by $G_{i}(t)$ the position of the center of mass at time $t$ of $\mathcal{B}_{i}(t)$, and $\theta_{i}(t)$ its rotation angle with respect to the rotation axis represented by the element $\vec{R}_{i}(t)$ on the unit sphere (if $d=2, \vec{R}_{i}(t)$ is a constant vector orthogonal to the plane where the motion takes place), so that

$$
x(t) \in \mathcal{B}_{i}(t) \Leftrightarrow \overrightarrow{G_{i} x}(t)=\exp \left(\theta_{i}(t)\left[\vec{R}_{i}(t) \wedge\right]\right) \overrightarrow{G_{i} x}(0),
$$

where $\left[\vec{R}_{i}(t) \wedge\right]$ is the operator "exterior product". In order to be more explicit, we rewrite (1) as follows: in $2 \mathrm{D}$

$$
x(t) \in \mathcal{B}_{i}(t) \Leftrightarrow \overrightarrow{G_{i} x}(t)=\left(\begin{array}{cc}
\cos \left(\theta_{i}(t)\right) & -\sin \left(\theta_{i}(t)\right) \\
\sin \left(\theta_{i}(t)\right) & \cos \left(\theta_{i}(t)\right)
\end{array}\right) \overrightarrow{G_{i} x}(0),
$$

\footnotetext{
Keywords and phrases. Navier-Stokes, fluid structure interaction.

1 Ceremade, Université Paris Dauphine, 75775 Paris Cedex 16, France.

2 Laboratoire ASCI, Bât. 506, Université Paris Sud, 91405 Orsay, Cedex, France et ONERA, 29 avenue Division Leclerc, 92322 Châtillon, France.
} 
whereas in $3 \mathrm{D}$

$$
x(t) \in \mathcal{B}_{i}(t) \Leftrightarrow \overrightarrow{G_{i} x}(t)=M_{x}(t) M_{y}(t) M_{z}(t) \overrightarrow{G_{i} x}(0),
$$

where

$$
\begin{aligned}
& M_{x}(t)=\left(\begin{array}{ccc}
1 & 0 & 0 \\
0 & \cos \left(\left(R_{i}\right)_{x} \theta_{i}(t)\right) & -\sin \left(\left(R_{i}\right)_{x} \theta_{i}(t)\right) \\
0 & \sin \left(\left(R_{i}\right)_{x} \theta_{i}(t)\right) & \cos \left(\left(R_{i}\right)_{x} \theta_{i}(t)\right)
\end{array}\right), \\
& M_{y}(t)=\left(\begin{array}{ccc}
\cos \left(\left(R_{i}\right)_{y} \theta_{i}(t)\right) & 0 & -\sin \left(\left(R_{i}\right)_{y} \theta_{i}(t)\right) \\
0 & 1 & 0 \\
\sin \left(\left(R_{i}\right)_{y} \theta_{i}(t)\right) & 0 & \cos \left(\left(R_{i}\right)_{y} \theta_{i}(t)\right)
\end{array}\right), \\
& M_{z}(t)=\left(\begin{array}{ccc}
\cos \left(\left(R_{i}\right)_{z} \theta_{i}(t)\right) & -\sin \left(\left(R_{i}\right)_{z} \theta_{i}(t)\right) & 0 \\
\sin \left(\left(R_{i}\right)_{z} \theta_{i}(t)\right) & \cos \left(\left(R_{i}\right)_{z} \theta_{i}(t)\right) & 0 \\
0 & 0 & 1
\end{array}\right),
\end{aligned}
$$

and where $R_{i x}, R_{i y}, R_{i z}$ are the three components of $\vec{R}_{i}(t)$ in a fixed given coordinate system. The NavierStokes equations are then set in $\Omega(t)=\Omega \backslash \cup_{i=1}^{N} \mathcal{B}_{i}(t)$ which is an unknown domain depending on time. The boundary $\Gamma_{0}$ of $\Omega$ and the boundaries $\partial \mathcal{B}_{i}$ of the various bodies are assumed to be regular enough in all what follows.

The equations for the fluid part are

$$
\begin{cases}\partial_{t} \mathbf{u}+(\mathbf{u} . \nabla) \mathbf{u}-\nu \Delta \mathbf{u}+\nabla p=\mathbf{f} & \text { in } \Omega(t) \\ \operatorname{div} \mathbf{u}=0 & \text { in } \Omega(t) \\ \mathbf{u}(t, .)=0 & \text { on } \Gamma_{0} \\ \mathbf{u}(t, x(t))=\mathbf{w}_{G_{i}}(t)+\frac{\mathrm{d}\left(\theta_{i} \vec{R}_{i}\right)}{\mathrm{d} t}(t) \wedge \overrightarrow{G_{i} x}(t) & \text { on } \partial \mathcal{B}_{i}(t) \\ \mathbf{u}(0, .)=\mathbf{u}_{0} & \text { in } \Omega(0),\end{cases}
$$

where $\mathbf{u}$ denotes the fluid velocity, $p$ its pressure, $\mathbf{w}_{G_{i}}$ the velocity of the center of mass; the applied exterior force $\mathbf{f}$ is given together with the initial velocity $\mathbf{u}_{0}$. For the structure part, recalling that for each point of the $i$ th body we have

so that

$$
\dot{x}(t)=\dot{G}_{i}(t)+\frac{\mathrm{d}}{\mathrm{d} t}(\theta \vec{R})(t) \wedge \overrightarrow{G_{i}}(t),
$$

$$
\ddot{x}(t)=\ddot{G}_{i}(t)+\frac{\mathrm{d}^{2}}{\mathrm{~d} t^{2}}(\theta \vec{R})(t) \wedge \overrightarrow{G_{i} x}(t)+\frac{\mathrm{d}}{\mathrm{d} t}(\theta \vec{R})(t) \wedge\left(\frac{\mathrm{d}}{\mathrm{d} t}(\theta \vec{R})(t) \wedge \overrightarrow{G_{i} x}(t)\right),
$$

and applying the conservation of linear and angular momentum we obtain

$$
\left\{\begin{array}{l}
m_{i} \frac{\mathrm{d} \mathbf{w}_{G_{i}}}{\mathrm{~d} t}=\int_{\partial \mathcal{B}_{i}(t)}\left(p-\nu\left(\nabla+\nabla^{T}\right) \mathbf{u}\right) \cdot \mathbf{n}_{i}(t) \mathrm{d} x, \\
J_{i} \frac{\mathrm{d}^{2} \theta_{i} \vec{R}_{i}}{\mathrm{~d} t^{2}}+\int_{\partial \mathcal{B}_{i}(t)} \rho_{i} \frac{\mathrm{d}}{\mathrm{d} t}\left(\theta_{i} \vec{R}_{i}\right)(t) \wedge\left(\frac{\mathrm{d}}{\mathrm{d} t}\left(\theta_{i} \vec{R}_{i}\right)(t) \wedge \overrightarrow{G_{i} x}(t)\right)= \\
\int_{\partial \mathcal{B}_{i}(t)} \overrightarrow{G_{i} x}(t) \wedge\left(\left(p-\nu\left(\nabla+\nabla^{T}\right) \mathbf{u}\right) \cdot \mathbf{n}_{i}(t)\right) \mathrm{d} x,
\end{array}\right.
$$


where $\mathbf{n}_{i}(t)$ denotes the exterior unit normal of $\mathcal{B}_{i}(t)$. Note that in the general $2 D$ and in the case of a $3 D$ sphere the last term on the left hand side cancels. The velocity $\mathbf{w}_{G_{i}}$ depends on the resulting stress coming from the fluid and the rotation velocity is determined by the resulting moment of the fluid forces.

We have to impose also initial conditions for the bodies

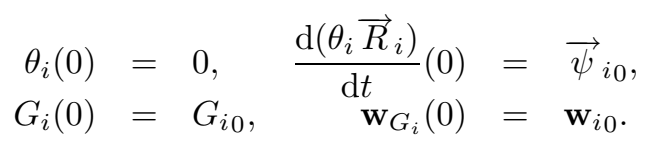

In order to ensure the well-posedness of the coupled problem, we impose, moreover, compatibility conditions over the initial conditions

$$
\operatorname{div} \mathbf{u}_{0}=0,\left.\quad \mathbf{u}_{0}\right|_{\Gamma_{0}}=0,\left.\quad \mathbf{u}_{0}\right|_{\partial \mathcal{B}_{i}}=\mathbf{w}_{i 0}+\left.\vec{\psi}_{i_{0}} \wedge \overrightarrow{G_{i} x}(0)\right|_{\partial \mathcal{B}_{i}} .
$$

Remark 1. The standard compatibility condition between the fluid incompressibility and the boundary conditions is satisfied here. This condition is derived from the fact that divu $=0$ in $\Omega(t)$ implies necessarily that $\int_{\partial \Omega(t)} \mathbf{u} \cdot \mathbf{n}=0$, i.e. here

$$
\sum_{i=1}^{N} \int_{\partial \mathcal{B}_{i}(t)} \mathbf{u} \cdot \mathbf{n}_{i}(t)=0
$$

But $\mathbf{u}$ over $\partial \mathcal{B}_{i}(t)$ is equal to a velocity associated to a rigid body motion which is the sum of a translation velocity and a rotation velocity. Thus (8) is satisfied.

We have an unsteady coupled problem. Since the fluid domain is an unknown of the problem and depends on time, we rewrite, in a first step, the Navier-Stokes equations on $\Omega(0)$, by using the Lagrangian variables. We denote by $\mathbf{v}$ the Lagrangian velocity of the fluid and set:

$$
\chi_{\mathbf{v}}(t, \xi)=\xi+\int_{0}^{t} \mathbf{v}(s, \xi) \mathrm{d} s .
$$

So that,

$$
\mathbf{u}\left(t, \chi_{\mathbf{v}}(t, \xi)\right)=\mathbf{v}(t, \xi)
$$

Making use of this change of variables we obtain — at least formally -

$$
\begin{cases}\partial_{t} \mathbf{v}-\nu\left(\nabla_{\mathbf{v}}\right)^{2} \mathbf{v}+\nabla_{\mathbf{v}} q=\mathbf{f} \circ \chi_{\mathbf{v}} & \text { in } \Omega(0) \\ \nabla_{\mathbf{v}} \cdot \mathbf{v}=0 & \text { in } \Omega(0) \\ \mathbf{v}=0 & \text { on } \Gamma_{0} \\ \mathbf{v}=\mathbf{w}_{G_{i}}+\frac{\mathrm{d}\left(\exp \left(\theta_{i}\left[\vec{R}_{i} \wedge\right]\right)\right)}{\mathrm{d} t} \overrightarrow{G_{i} x}(0) & \text { on } \partial \mathcal{B}_{i} \\ \mathbf{v}(0)=\mathbf{u}_{0} & \text { in } \Omega(0),\end{cases}
$$

where we set $q(t, \xi)=p\left(t, \chi_{\mathbf{v}}(t, \xi)\right)$. We mean by $\mathbf{f} \circ \chi_{\mathbf{v}}$ the function defined by $\mathbf{f}\left(t, \chi_{\mathbf{v}}(t, \xi)\right)$. The operator $\nabla_{\mathbf{v}}$ denotes the operator $\operatorname{cof}\left(\nabla \chi_{\mathbf{v}}\right) . \nabla$. We have used the fact that $\operatorname{det} \nabla \chi_{\mathbf{v}}=1$, which comes from the fluid incompressibility and from the relation

$$
\frac{\partial}{\partial t} \operatorname{det} \nabla \chi_{\mathbf{v}}=\operatorname{div} \mathbf{u} \circ \chi_{\mathbf{v}} \operatorname{det} \nabla \chi_{\mathbf{v}} .
$$


For the proof of the last equality we refer to [9] Chapter 1 pp. 26-27, or [17]. We note that the convection term $(\mathbf{u} . \nabla) \mathbf{u}$ disappears due to

$$
\partial_{t} \mathbf{v}(t, \xi)=\left(\partial_{t} \mathbf{u}+(\mathbf{u} \cdot \nabla) \mathbf{u}\right)\left(t, \chi_{\mathbf{v}}(t, \xi)\right) .
$$

We can also rewrite the structure equation with the help of the new unknowns, we get

$$
\left\{\begin{array}{l}
m_{i} \frac{\mathrm{d} \mathbf{w}_{G_{i}}}{\mathrm{~d} t}=\int_{\partial \mathcal{B}_{i}}\left(q-\nu\left(\nabla_{\mathbf{v}}+\nabla_{\mathbf{v}}{ }^{T}\right) \mathbf{v}\right) \operatorname{cof}\left(\nabla \chi_{\mathbf{v}}\right) \cdot \mathbf{n}_{i} \mathrm{~d} x \\
J_{i} \frac{\mathrm{d}^{2} \theta_{i} \vec{R}_{i}}{\mathrm{~d} t^{2}}+\int_{\partial \mathcal{B}_{i}} \rho_{i} \frac{\mathrm{d}}{\mathrm{d} t}\left(\theta_{i} \vec{R}_{i}\right)(t) \wedge\left(\frac{\mathrm{d}}{\mathrm{d} t}\left(\theta_{i} \vec{R}_{i}\right)(t) \wedge\left(\exp \left(\theta_{i}\left[\overrightarrow{R_{i} \wedge}\right]\right) \overrightarrow{G_{i} x}(0)\right)\right) \mathrm{d} x= \\
\int_{\partial \mathcal{B}_{i}}\left(\exp \left(\theta_{i}\left[\vec{R}_{i} \wedge\right]\right) \overrightarrow{G_{i} x}(0)\right) \wedge\left[\left(q-\nu\left(\nabla_{\mathbf{v}}+\nabla_{\mathbf{v}}{ }^{T}\right) \mathbf{v}\right) \cdot\left(\operatorname{cof}\left(\nabla \chi_{\mathbf{v}}\right) \cdot \mathbf{n}_{i}\right)\right] \mathrm{d} x,
\end{array}\right.
$$

where $\mathbf{n}_{i}$ denotes the exterior unit normal to $\mathcal{B}_{i}$.

We are looking for a solution of $(10,11)$ and are going to prove an existence result locally in time in some Sobolev spaces, that have first been used by J. T. Beale in a similar context. We shall often use their interpolation properties. In what follows, we do not distinguish the space of scalar-valued functions $H^{s}(\Omega, \mathbb{R})$ from the space of vector-valued functions $H^{s}\left(\Omega, \mathbb{R}^{d}\right)$, we will simply write $H^{s}(\Omega)$. If $X$ is an Hilbert space, $H^{s}(0, T ; X)$ denotes the space functions $H^{s}$ with value in $X$. Because the time interval $(0, T)$ will be small, we have to be careful about the dependence of the various estimates on $T$. Thus, we make an explicit choice of the norm associated to spaces of type $H^{s}(0, T ; X)$. For $0<s<1$, we define $H^{s}(0, T ; X)$ as the domain of the operator $\Lambda^{s / 2}$ where $\Lambda=\left(1-\partial_{t}^{2}\right)$ and $D(\Lambda)=\left\{v \in H^{2}(0, T ; X) ; \partial_{t} v(0)=\partial_{t} v(T)=0\right\}$. This choice enables us to have the following property: let $Y$ and $Z$ be two Hilbert spaces such that $Z \subset Y$ and $Z$ is supposed to be dense in $Y$, assume that for all $T$, we have a continuous operator $A(T)$ from $H^{0}(0, T ; X)$ to $Y$ and from $H^{1}(0, T ; X)$ to $Z$ with a constant of continuity independent of $T$. Then $A(T)$ maps $H^{s}(0, T ; X)$ to the interpolated space $[Z, Y]_{1-s}$, $0 \leq s \leq 1$ with a continuity constant independent of $T$ (see Th. 1.5.1 [14]). For $m<s<m+1$, where $m$ is an integer, we define $H^{s}(0, T ; X)$ as the space of functions such that $\partial_{t}^{m} v \in H^{s-m}(0, T ; X)$. We set for $T>0$,

$$
K_{T}^{r}(\Omega)=L^{2}\left(0, T ; H^{r}(\Omega)\right) \cap H^{r / 2}\left(0, T ; L^{2}(\Omega)\right) .
$$

The main result is the following:

Theorem 1. Let $r$ be a real number, $1<r<3 / 2$. We assume that $\mathbf{u}_{0} \in H^{r+1}(\Omega(0))$, $\mathbf{f}$ is sufficiently smooth and that the mass and the moment of inertia of the bodies are sufficiently large, then there exists a time $T_{1}>0$ depending on $\Omega(0),\left\|\mathbf{u}_{0}\right\|_{H^{r+1}(\Omega(0))}, \mathbf{w}_{i 0}, \vec{\psi}_{i 0}$ and $\mathbf{f}$ such that the problem $(10,11)$ has a unique solution with $\mathbf{u} \in K_{T_{1}}^{r+2}(\Omega(0)), \nabla q \in K_{T_{1}}^{r}(\Omega(0)), \mathbf{w}_{G_{i}} \in H^{r / 2+1}\left(0, T_{1}\right)$ and $\left(\theta_{i} \vec{R}_{i}\right) \in H^{r / 2+2}\left(0, T_{1}\right)$.

Remark 2. As noted in [4] the real number $r$ has to be large enough in order to define and estimate the nonlinear terms which appear in the Lagrangian formulation of the fluid equations and also in order that the solution in the Lagrangian variables can be transformed into a solution of the original problem, i.e. where the fluid equations are written in the eulerian variables. Moreover, as the value of $r$ increases, more compatibility conditions should be imposed on the data of the problem (initial conditions, forces) to obtain a solution in the spaces we choose. This is the reason why $r$ is less than $3 / 2$. An alternative could then be to use weights in time as in [13].

In order to prove Theorem 1, we are going, in a first step, to study a fluid problem with a given velocity over $\partial \mathcal{B}_{i}$. For such equations we prove that there exists a smooth solution with the help of a fixed point theorem (contraction mapping principle). The ideas are the same that one can find in the papers $[2-4,16,17]$ where the authors have studied the solvability of the Navier-Stokes equations with free boundary in bounded or unbounded domains. Their approach is the following: the equations are rewritten in Lagrangian coordinates and it is shown that solutions for the initial value problem exist locally in time, in smooth functions spaces, that is to say the same kind of spaces we use here [2-4], or spaces of $W^{1, p}$-type with $p$ bigger than the spatial dimension [17], 
or in Hölder spaces [16]. Section 2 is devoted to the study of the fluid equations, and contains some standard lemmas which will be useful (Sect. 2.1) and the study of the linearised equations (the homogeneous case and inhomogeneous one). We obtain existence and regularity result for the linear problems. The existence of solutions for the nonlinear problem is proven, for a small enough time (Sect. 2.3). The proof is based on the estimates of the nonlinear terms and the contraction mapping principle. We have then to recouple the equations in order to obtain a solution of the original interaction problem (Sect. 3).

\section{Study OF THE FLUID PROBLEM}

We consider the following equations:

$$
\begin{cases}\partial_{t} \mathbf{v}-\nu\left(\nabla_{\mathbf{v}}\right)^{2} \mathbf{v}+\nabla_{\mathbf{v}} q=\mathbf{f} \circ \chi_{\mathbf{v}} & \text { in } \Omega(0) \\ \nabla_{\mathbf{v}} \cdot \mathbf{v}=0 & \text { in } \Omega(0) \\ \mathbf{v}=0 & \text { on } \Gamma_{0} \\ \mathbf{v}=\mathbf{v}_{b i} & \text { on } \partial \mathcal{B}_{i} \\ \mathbf{v}(0)=\mathbf{u}_{0} & \text { in } \Omega(0)\end{cases}
$$

where $\mathbf{v}_{b i}$ is given in $H^{r / 2+1}\left(0, T_{0} ; H^{l}\left(\partial \mathcal{B}_{i}\right)\right), l$ will be chosen latter $\left(T_{0}\right.$ is some strictly positive time) and $\mathbf{v}_{b i}$ verifies the following compatibility condition

$$
\sum_{i=1}^{N} \int_{\partial \mathcal{B}_{i}} \mathbf{v}_{b i} \cdot \operatorname{cof} \nabla \chi_{\mathbf{v}} \cdot \mathbf{n}_{i}=0
$$

which comes from $\int_{\Omega(0)} \nabla_{\mathbf{v}} \cdot \mathbf{v}=0$ by integration by parts. Note that the vector $\operatorname{cof} \nabla \chi_{\mathbf{v}} \cdot \mathbf{n}_{i}$ depends only on the tangential derivatives of $\mathbf{v}$ along $\partial \mathcal{B}_{i}$, thus only involves $\mathbf{v}_{b i}$. Note also that each term in this sum vanishes automatically for velocities $\mathbf{v}_{b i}$ associated with rigid body motions, since rigid body motions are incompressible.

Theorem 2. Let $r$ be a real number, $1<r<3 / 2$. We suppose that $\mathbf{u}_{0} \in H^{r+1}(\Omega(0))$, and that its trace on the boundary $\partial \mathcal{B}_{i}$ is regular enough, say belongs to $H^{l}\left(\partial \mathcal{B}_{i}\right)$ with $l \geq r+3 / 2$. We consider $\mathbf{v}_{\text {bi }} \in$ $H^{r / 2+1}\left(0, T_{0} ; H^{l}\left(\partial \mathcal{B}_{i}\right)\right)$, such that (13) is satisfied and we suppose that $\mathbf{f}$ is smooth enough (for instance $\left.C^{\infty}\right)$. We suppose, moreover, that

$$
\operatorname{div} \mathbf{u}_{0}=0,\left.\quad \mathbf{u}_{0}\right|_{\Gamma_{0}}=0
$$

then there exists $T_{1}>0$ depending on $\Omega(0),\left\|\mathbf{u}_{0}\right\|_{H^{r+1}(\Omega(0))},\left.\mathbf{u}_{0}\right|_{\partial \mathcal{B}_{i}},\left\|\mathbf{v}_{b i}\right\|_{H^{r / 2+1}\left(0, T_{0} ; H^{l}\left(\partial \mathcal{B}_{i}\right)\right)}$ and $\mathbf{f}$ such that the problem (12) has a unique solution with $\mathbf{v} \in K_{T_{1}}^{r+2}(\Omega(0)), \nabla q \in K_{T_{1}}^{r}(\Omega(0))$.

This theorem will be proven in several steps. First of all we study the linearised system associated with (12) and in particular we prove the existence of a smooth solution, first for the homogeneous equations and next we extend the result for the fully inhomogeneous problem. We then estimate the nonlinear terms, which are small for a small enough time, and we apply the contraction mapping theorem in order to obtain a solution of the original fluid problem with a given velocity on the boundary. We follow here closely the paper of J.T. Beale [4] and adapt carefully each step of his proof to our context. The main difference lies in the type of boundary conditions.

\subsection{Preliminary results}

In this subsection, we give some classical lemmas and theorems which are useful for our purpose, $\Omega$ will denote here any smooth enough domain regardless of the previous section.

Theorem 3. i) Suppose that $1 / 2<r \leq 5$. The mapping $v \mapsto \partial_{n}^{j} v$ is a bounded operator from $K_{T}^{r}(\Omega)$ into the space $K_{T}^{r-j-1 / 2}(\partial \Omega)$, where $j$ is an integer with $0 \leq j<r-1 / 2$. The mapping $v \mapsto \partial_{t}^{k} v(., 0)$ is also a bounded 
operator from $K_{T}^{r}(\Omega)$ into $H^{r-2 k-1}(\Omega)$, if $0 \leq k<\frac{(r-1)}{2}$.

ii) Suppose that $1 / 2<r<5, r \neq 1, r \neq 3$ and $r-1 / 2$ is not an integer. Let

$$
W^{r}=\prod_{0 \leq j<r-1 / 2} K_{T}^{r-j-1 / 2}(\partial \Omega) \times \prod_{0 \leq k<\frac{(r-1)}{2}} H^{r-2 k-1}(\Omega),
$$

and let $W_{0}^{r}$ the subspace of $W^{r}$ consisting of $\left(a_{j}, w_{k}\right)$ so that (when $r>3 / 2$ )

$$
\partial_{t}^{k} a_{j}(x, 0)=\partial_{n}^{j} w_{k}(x), \quad x \in \partial \Omega \quad \text { for } j+2 k<r-3 / 2 .
$$

Then the traces of i) sum up to a bounded operator from $K_{T}^{r}(\Omega)$ onto $W_{0}^{r}$; this operator has a bounded inverse.

For the proof of this theorem we refer to Chapter 4 of [14].

Lemma 1. Let $X$ be a Hilbert space.

i) For $s \geq 0$, there exists a bounded extension operator from $H^{s}(0, T ; X)$ into $H^{s}(0, \infty ; X)$.

ii) For $0 \leq s<7 / 2, s-1 / 2$ not an integer, there exists an extension operator from the subspace $\left\{v \in H^{s}(0, T ; X) /\right.$ $\partial_{t}^{k} v(0)=0$ pour $\left.0 \leq k<s-1 / 2\right\}$ into $H^{s}(0, \infty ; X)$ with a norm bounded independently of $T$.

Proof. For the first part, we refer to the Theorem 2.2 page 17 of [14]. For $i i)$, if $s$ is an integer and $v$ belongs to the subspace of $H^{s}(0, T ; X)$ introduced in the lemma, we extend $v$ by 0 for $t<0$ and for $t>0$ we set:

$$
v(t+T)=10 v(T-2 t)-15 v(T-3 t)+6 v(T-4 t) .
$$

The extension operator we built has the desired properties. In the case where $s$ is not an integer the results follows by interpolation.

Remark 3. A more general lemma can be stated that deals with the cases $s \geq 7 / 2$. Different linear combinaisons than (14) which match more derivatives have then to be considered.

Lemma 2. Let $0 \leq r \leq 6$.

i) The identity is a bounded operator from $K_{T}^{r}(\Omega)$ into $H^{p}\left(0, T ; H^{r-2 p}(\Omega)\right)$ for $p \leq r / 2$.

ii) If $r$ is not an odd integer, the restriction of this operator to the subspace with $\partial_{t}^{k} v(0)=0$ for $0 \leq k<\frac{r-1}{2}$ is bounded independently of $T$.

Proof. The first part $i$ ) can be easily derived by extending the functions to $\mathbb{R}$ (with a norm maybe depending on $T$ ) and by using the Fourier transform with respect to time. For $i i$ ) we apply the previous lemma with $s=r / 2$.

Lemma 3. Let $T_{0}>0$ be arbitrary and choose $T$ such that $0<T \leq T_{0}$. For any $v \in L^{2}(0, T ; X)$ we define $V \in H^{1}(0, T ; X)$ by

$$
V(t)=\int_{0}^{t} v(\tau) \mathrm{d} \tau
$$

i) For $0 \leq s<1 / 2$, and $0 \leq \varepsilon \leq 1$ the operator $v \mapsto V$ is a bounded operator from $H^{s}(0, T ; X)$ into $H^{s+1-\varepsilon}(0, T ; X)$ and satisfies

$$
\|V\|_{H^{s+1-\varepsilon}(0, T ; X)} \leq C_{0} T^{\varepsilon}\|v\|_{H^{s}(0, T ; X)},
$$

for a constant $C_{0}$ independent of $T \leq T_{0}$.

ii) For $1 / 2<s \leq 1$ the estimate (15) is still satisfied, if furthermore $v(0)=0$. 
Proof. The Cauchy-Schwartz inequality easily leads to $\|V(t)\|_{X} \leq t^{1 / 2}\|v\|_{L^{2}(0, T ; X)}$ then to $\|V\|_{L^{2}(0, T ; X)} \leq$ $T\|v\|_{L^{2}(0, T ; X)}$ and then $\|V\|_{H^{1}(0, T ; X)} \leq(T+1)\|v\|_{L^{2}(0, T ; X)} \leq C\|v\|_{L^{2}(0, T ; X)}$. Hence, recalling the convexity property of Sobolev norms:

$$
\|V\|_{H^{1-\varepsilon}(0, T ; X)} \leq\|V\|_{H^{1}(0, T ; X)}^{1-\varepsilon}\|V\|_{L^{2}(0, T ; X)}^{\varepsilon},
$$

we have

$$
\|V\|_{H^{1-\varepsilon}(0, T ; X)} \leq C T^{\varepsilon}\|v\|_{L^{2}(0, T ; X)}
$$

Moreover, if $v \in H^{1}(0, T ; X)$ with $v(0)=0$ then $v(t)=\int_{0}^{t} \partial_{t} v(\tau) \mathrm{d} \tau$, and thus we check

$$
\begin{gathered}
\|v\|_{L^{2}(0, T ; X)} \leq T\left\|\partial_{t} v\right\|_{L^{2}(0, T ; X)}, \\
\|V\|_{H^{1}(0, T ; X)} \leq T\|v\|_{H^{1}(0, T ; X)}, \\
\|V\|_{H^{2}(0, T ; X)} \leq C\|v\|_{H^{1}(0, T ; X)} .
\end{gathered}
$$

Therefore by convexity

$$
\|V\|_{H^{2-\varepsilon}(0, T ; X)} \leq C T^{\varepsilon}\|v\|_{H^{1}(0, T ; X)} .
$$

We can now interpolate between the two estimates (16), (17), and we obtain the desired conclusion.

Lemma 4. i) Let $r>d / 2$ and $r \geq s \geq 0$. If $v \in H^{r}(\Omega)$ and $w \in H^{s}(\Omega)$ then $v w \in H^{s}(\Omega)$ with $\|v w\|_{H^{s}(\Omega)} \leq$ $C\|v\|_{H^{r}(\Omega)}\|w\|_{H^{s}(\Omega)}$.

ii) If $v \in H^{r}(\Omega)$ with $r>d / 2$ and if $w$ belongs to the dual space of $H^{1}(\Omega)$ then $v w$ is defined in $\left(H^{1}(\Omega)\right)^{\prime}$ and $\|v w\|_{\left(H^{1}(\Omega)\right)^{\prime}} \leq C\|v\|_{H^{r}(\Omega)}\|w\|_{\left(H^{1}(\Omega)\right)^{\prime}}$.

iii) If $v, w \in H^{1}(\Omega)$ then $v w \in L^{2}(\Omega)$ and $\|v w\|_{L^{2}(\Omega)} \leq C\|v\|_{H^{1}(\Omega)}\|w\|_{H^{1}(\Omega)}$.

iv) If $v \in H^{1}(\Omega)$ and $w \in L^{2}(\Omega)$ then $v w \in\left(H^{1}(\Omega)\right)^{\prime}$ with $\|v w\|_{\left(H^{1}(\Omega)\right)^{\prime}} \leq C\|v\|_{H^{1}(\Omega)}\|w\|_{L^{2}(\Omega)}$.

Proof. For the first part $i$ ), the case where $s=r$ is standard and relies on the fact that $H^{r}(\Omega)$ is an algebra for $r>d / 2$. The case $s=0$ comes from the Sobolev continuous embedding of $H^{r}(\Omega)$ into $L^{\infty}(\Omega)$. The other cases follow from hilbertian interpolation, by considering the multiplication by $w$ as a continuous linear operator. For iii), it suffices to use Sobolev inequality (see $[1,5]$ ). For the second point, we consider the multiplication on $\left(H^{1}(\Omega)\right)^{\prime}$ by an element of $H^{r}(\Omega)$ as the adjoint of the multiplication on $H^{1}(\Omega)$ and we use the first assertion. Similarly, $i v$ ) follows from $i i i)$.

Lemma 5. Let $X, Y, Z$ be three Hilbert spaces and $m: X \times Y \mapsto Z$ be a bounded, bilinear application.

i) If $v \in H^{s}(0, T ; X)$ and $w \in H^{s}(0, T ; Y)$ where $s>1 / 2$ then $m(v, w) \in H^{s}(0, T ; Z)$ and $\|m(v, w)\|_{H^{s}(0, T ; Z)} \leq$ $C\|v\|_{H^{s}(0, T ; X)}\|w\|_{H^{s}(0, T ; Y)}$.

ii) If $s<7 / 2$ and $v, w$ satisfy also the additional conditions $\partial_{t}^{k} v(0)=\partial_{t}^{k} w(0)=0,0 \leq k<s-1 / 2$, and $s-1 / 2$ is not an integer, then the constant $C$ is independent of $T>0$.

Proof. For $i$ ), we use the fact that, in one dimension, $H^{s}$ with $s>1 / 2$ is an algebra. For $i i$ ), we use Lemma 1 . 


\subsection{The linearised problem}

For the sake of simplicity, we will consider only one rigid body $\mathcal{B}$, but the analysis is the same with a finite number of moving rigid bodies. The linearised problem associated to (12) is:

$$
\begin{cases}\partial_{t} \mathbf{v}-\nu \Delta \mathbf{v}+\nabla q=\mathbf{f} & \text { in } \Omega(0), \\ \operatorname{div} \mathbf{v}=\rho & \text { in } \Omega(0), \\ \mathbf{v}=\mathbf{v}_{b} & \text { on } \partial \mathcal{B}, \\ \mathbf{v}=0 & \text { on } \Gamma_{0} \\ \mathbf{v}(0)=\mathbf{u}_{0} & \text { in } \Omega(0)\end{cases}
$$

where $\mathbf{f}, \rho, \mathbf{v}_{b}$ and $\mathbf{u}_{0}$ are data which satisfy the following compatibility conditions:

$$
\int_{\Omega(0)} \rho=\int_{\partial \mathcal{B}} \mathbf{v}_{b} \cdot \mathbf{n}, \quad \mathbf{u}_{0}=0 \quad \text { on } \quad \Gamma_{0}, \operatorname{div} \mathbf{u}_{0}=\rho(t=0) \text { in } \Omega(0),\left.\quad \mathbf{u}_{0}\right|_{\partial \mathcal{B}}=\mathbf{v}_{b}(t=0) .
$$

\subsubsection{The homogeneous linearised problem}

In a first step we study the homogeneous linearised problem (existence and regularity of solutions).

$$
\begin{cases}\partial_{t} \mathbf{v}-\nu \Delta \mathbf{v}+\nabla q=\mathbf{f} & \text { in } \Omega(0), \\ \operatorname{div} \mathbf{v}=0 & \text { in } \Omega(0), \\ \mathbf{v}=0 & \text { on } \partial \mathcal{B} \cup \Gamma_{0}, \\ \mathbf{v}(0)=0 & \text { in } \Omega(0)\end{cases}
$$

We call $\Pi$ the $L^{2}(\Omega(0))$-projection on $H$ defined by

$$
H \stackrel{\text { def }}{=}\left\{\mathbf{w} \in L^{2}(\Omega(0)) / \operatorname{div} \mathbf{w}=0 \text { in } \Omega(0), \quad \mathbf{w} \cdot \mathbf{n}=0 \text { on } \partial \Omega(0)\right\} .
$$

A standard result makes precise the orthogonal $H^{\perp}$ of $H$ in $L^{2}(\Omega(0))$ :

$$
H^{\perp}=\left\{\nabla p, \quad p \in H^{1}(\Omega(0))\right\} .
$$

Moreover, we have

Lemma 6. i) $\Pi$ is a bounded operator on $H^{s}(\Omega(0))$.

ii) $\Pi$ is also bounded on $K_{T}^{s}(\Omega(0))$, with a norm bounded independent of $T$.

Proof. $i)$ Suppose that $s \geq 1$. Let $\mathbf{v} \in H^{s}(\Omega(0))$. By definition of $\Pi$ and from the characterisation of $H^{\perp}$ $(I-\Pi) \mathbf{v}=\nabla \chi$, where $\chi$ is defined by

$$
(\nabla \chi, \nabla \phi)=(\mathbf{v}, \nabla \phi), \forall \phi \in H^{1}(\Omega(0))
$$

Therefore, $\chi$ is a weak solution of

$$
\begin{aligned}
& \Delta \chi=\nabla \cdot \mathbf{v} \quad \text { in } \Omega(0), \\
& \frac{\partial \chi}{\partial \mathbf{n}}=\mathbf{v} \cdot \mathbf{n} \quad \text { on } \partial \Omega(0) \text {. }
\end{aligned}
$$

Taking into account the regularity properties of the Laplace equation with Neumann boundary conditions, and since by assumption $\partial \Omega(0)$ is smooth, we derive that $\chi \in H^{s+1}(\Omega(0))$ and we have the following estimate

$$
\|\chi\|_{H^{s+1}(\Omega(0))} \leq C\|\mathbf{v}\|_{H^{s}(\Omega(0))} .
$$

Thus $I-\Pi$, and consequently $\Pi$ are bounded operators on $H^{s}(\Omega(0))$ for $s \geq 1$. By definition $\left.i\right)$ is satisfied for $s=0$. The other cases follow by interpolation. 
ii) Let $s$ be an even integer, the properties come from the first assertion because the projection $\Pi$ commutes with the time derivative. The other cases follow by interpolation.

We state now the proposition in the homogeneous case.

Proposition 1. Suppose that $\mathbf{f} \in K_{T}^{r}(\Omega(0))$ with $0 \leq r \leq 2, r \neq 1$ and suppose that $\Pi \mathbf{f}(0)=0$ if $r>1$, then there exits a unique solution $(\mathbf{v}, p)$ of the problem (20) with $\mathbf{v} \in K_{T}^{r+2}(\Omega(0)), \nabla p \in K_{T}^{r}(\Omega(0))$ and $\int_{\Omega(0)} p=0$. Furthermore,

$$
\|\mathbf{v}\|_{K_{T}^{r+2}(\Omega(0))}+\|\nabla p\|_{K_{T}^{r}(\Omega(0))} \leq C\|\mathbf{f}\|_{K_{T}^{r}(\Omega(0))}
$$

where $C$ denotes a constant independent of $T \leq T_{0}$.

Proof. The proof is separated in several steps. We prove the proposition first for $r=0$, then for $r=2$.

When $\mathbf{f} \in L^{2}\left(0, T ; L^{2}(\Omega(0))\right.$ ) (this corresponds to the case $\left.r=0\right)$, it is shown in [10], or [19] Chapter III, p. 267, that there exists a unique solution $(\mathbf{v}, p)$ of $(20)$ with $\mathbf{v} \in L^{2}\left(0, T ; H^{2}(\Omega(0))\right) \cap H^{1}\left(0, T ; L^{2}(\Omega(0))\right)$, $p \in L^{2}\left(0, T ; H^{1}(\Omega(0))\right)$ and $\int_{\Omega(0)} p=0$. Moreover, we have

$$
\|\mathbf{v}\|_{L^{2}\left(0, T ; H^{2}(\Omega(0))\right) \cap H^{1}\left(0, T ; L^{2}(\Omega(0))\right)}+\|p\|_{L^{2}\left(0, T ; H^{1}(\Omega(0))\right)} \leq C\|\mathbf{f}\|_{L^{2}\left(0, T ; L^{2}(\Omega(0))\right)},
$$

where $C$ is a constant independent of the time $T \leq T_{0}$.

In the case $r=2$, we consider $\mathbf{f} \in L^{2}\left(0, T ; H^{2}(\Omega(0))\right) \cap H^{1}\left(0, T ; L^{2}(\Omega(0))\right)=K_{T}^{2}(\Omega(0))$ with the additional assumption $\Pi \mathbf{f}(0)=0$.

First we suppose that $\mathbf{f}(0)=0$.

Let $(\mathbf{z}, q)$ be the solution of

$$
\begin{cases}\partial_{t} \mathbf{z}-\nu \Delta \mathbf{z}+\nabla q=\partial_{t} \mathbf{f} & \text { in } \Omega(0), \\ \operatorname{div} \mathbf{z}=0 & \text { in } \Omega(0), \\ \mathbf{z}=0 & \text { on } \partial \mathcal{B} \cup \Gamma_{0}, \\ \mathbf{z}(0)=0 & \text { in } \Omega(0) .\end{cases}
$$

We remark that $\partial_{t} \mathbf{f} \in L^{2}\left(0, T ; L^{2}(\Omega(0))\right)$, and so we obtain a solution $(\mathbf{z}, q)$ belonging to $K_{T}^{2}(\Omega(0)) \times L^{2}\left(0, T ; H^{1}(\Omega(0))\right)$. We set

$$
\mathbf{v}=\int_{0}^{t} \mathbf{z}, \quad p=\int_{0}^{t} q .
$$

Then, $(\mathbf{v}, p)$ is a solution of

$$
\begin{cases}\partial_{t} \mathbf{v}-\nu \Delta \mathbf{v}+\nabla p=\mathbf{f} & \text { in } \Omega(0), \\ \operatorname{div} \mathbf{v}=0 & \text { in } \Omega(0), \\ \mathbf{v}=0 & \text { on } \partial \mathcal{B} \cup \Gamma_{0} \\ \mathbf{v}(0)=0 & \text { in } \Omega(0)\end{cases}
$$

If we choose the average of $q$ equal to zero then $\int_{\Omega(0)} p=0$.

This solution verifies

$$
\partial_{t} \mathbf{v}=\mathbf{z} \in K_{T}^{2}(\Omega(0)), \quad \partial_{t} p=q \in L^{2}\left(0, T ; H^{1}(\Omega(0))\right),
$$

and, thanks to the previous step

$$
\left\|\partial_{t} \mathbf{v}\right\|_{K_{T}^{2}(\Omega(0))}+\left\|\partial_{t} p\right\|_{L^{2}\left(0, T ; H^{1}(\Omega(0))\right)} \leq C\left\|\partial_{t} \mathbf{f}\right\|_{L^{2}\left(0, T ; L^{2}(\Omega(0))\right)}
$$


Moreover, we get

$$
\begin{cases}-\nu \Delta \mathbf{v}+\nabla p=f-\partial_{t} \mathbf{v} \in L^{2}\left(0, T ; H^{2}(\Omega(0))\right), & \\ \operatorname{div} \mathbf{v}=0 & \text { in } \Omega(0), \\ \mathbf{v}=0 & \text { on } \partial \Omega(0) .\end{cases}
$$

Using the regularities properties of the steady state Stokes equations with $\Omega(0)$ smooth (see [10] Th. I. 5.4, p. 88), we deduce that $\mathbf{v} \in L^{2}\left(0, T ; H^{4}(\Omega(0))\right), p \in L^{2}\left(0, T ; H^{3}(\Omega(0))\right)$ and

$$
\|\mathbf{v}\|_{L^{2}\left(0, T ; H^{4}(\Omega(0))\right)}+\|p\|_{L^{2}\left(0, T ; H^{3}(\Omega(0))\right)} \leq C\left[\left\|\partial_{t} \mathbf{v}\right\|_{L^{2}\left(0, T ; H^{2}(\Omega(0))\right)}+\|\mathbf{f}\|_{L^{2}\left(0, T ; H^{2}(\Omega(0))\right)}\right] \leq C\|\mathbf{f}\|_{K_{T}^{2}(\Omega(0))} .
$$

Then we have $(\mathbf{v}, p)$ solution of $(20)$ with $\mathbf{f}(0)=0$, and such that

$$
\mathbf{v} \in K_{T}^{4}(\Omega(0)), \quad \nabla p \in K_{T}^{2}(\Omega(0))
$$

with the estimate

$$
\|\mathbf{v}\|_{K_{T}^{4}(\Omega(0))}+\|\nabla p\|_{K_{T}^{2}(\Omega(0))} \leq C\|\mathbf{f}\|_{K_{T}^{2}(\Omega(0))},
$$

where $C$ denotes a constant independent of $T$.

When $0<r<2$ and $r \neq 1$, we obtain the desired result by interpolating the spaces $L^{2}\left(0, T ; L^{2}(\Omega(0))\right)$ and $\left\{\mathbf{f} \in K_{T}^{2}(\Omega(0)) / \mathbf{f}(0)=0\right\}$. The interpolation between these two spaces faces no difficulty because we can extend in time the functions belonging to $\left\{\mathbf{f} \in K_{T}^{2}(\Omega(0)) / \mathbf{f}(0)=0\right\}$ to $\mathbb{R}$ so that the norm of the extension is independent of $T$.

The next step is to consider a given force that satisfies $\Pi \mathbf{f}(0)=0$. We have $\mathbf{f}=\Pi \mathbf{f}+(I-\Pi) \mathbf{f}$. For $\Pi \mathbf{f}$ we apply the previous result. Then, there exists a couple $(\tilde{\mathbf{v}}, \tilde{p})$ which is solution of (20) with $\Pi \mathbf{f}$ as a data, such that $\tilde{\mathbf{v}} \in K_{T}^{r+2}(\Omega(0))$ and $\nabla \tilde{p} \in K_{T}^{r}(\Omega(0))$ and which satisfies

$$
\|\tilde{\mathbf{v}}\|_{K_{T}^{r+2}(\Omega(0))}+\|\nabla \tilde{p}\|_{K_{T}^{r}(\Omega(0))} \leq C\|\Pi \mathbf{f}\|_{K_{T}^{r}(\Omega(0))} .
$$

The second part $i$ ) of the Lemma 6 yields

$$
\|\tilde{\mathbf{v}}\|_{K_{T}^{r+2}(\Omega(0))}+\|\nabla \tilde{p}\|_{K_{T}^{r}(\Omega(0))} \leq C\|\mathbf{f}\|_{K_{T}^{r}(\Omega(0))}
$$

with a constant $C$ independent of the time $T$. On the other hand, as we already seen, $(I-\Pi) \mathbf{f}=\nabla \chi$. But $I-\Pi$ is a bounded operator whose norm is independent of $T$ and consequently $\|\nabla \chi\|_{K_{T}^{r}(\Omega(0))} \leq C\|\mathbf{f}\|_{K_{T}^{r}(\Omega(0))}$ $(C$ independent of $T)$. Setting $(\mathbf{v}, p)=(\tilde{\mathbf{v}}, \tilde{p}+\chi)$, we obtain a solution of $(20)$ associated to $\mathbf{f}$ which satisfies the desired estimates. This ends the proof.

\subsubsection{Inhomogeneous linearised problem}

We now extend the results of the last section to a more general problem: the inhomogeneous case. But, first of all, we start by making a remark, which will enable us to specify the spaces in which we will choose the data of the problem. If $\psi \in K_{T}^{r+2}(\Omega(0))$ with $\left.\psi\right|_{\Gamma_{0}}=0$ and $\left.\psi\right|_{\partial \mathcal{B}}=\mathbf{z} \in H^{r / 2+1}\left(0, T ; H^{l}(\partial \mathcal{B})\right)$ with $l \geq r+3 / 2$, then $\operatorname{div} \psi \in \hat{K}_{T}^{r}(\Omega(0))$, where $\hat{K}_{T}^{r}(\Omega(0))$ is defined by

$$
\hat{K}_{T}^{r}(\Omega(0)) \stackrel{\text { def }}{=} L^{2}\left(0, T ; H^{r+1}(\Omega(0))\right) \cap H^{1+r / 2}\left(0, T ;\left(H^{1}(\Omega(0))\right)^{\prime}\right) .
$$

Indeed, suppose first that $r$ is an even integer, $r \geq 0$, and $\mathbf{z}=0$. If $\psi$ is a smooth function, let us choose $\psi$ in $H^{s}\left(0, T ; H^{r+2}(\Omega(0))\right)$, where $\left.\psi\right|_{\Gamma_{0} \cup \partial \mathcal{B}}=0$. Then,

$$
\forall \phi \in H^{1}(\Omega(0)), \forall j \leq 1+r / 2 \quad\left(\partial_{t}^{j}(\operatorname{div} \psi), \phi\right)=-\left(\partial_{t}^{j} \psi, \nabla \phi\right),
$$


where (.,.) denotes the $L^{2}(\Omega(0))$-inner product. Thus for each $t$

$$
\left\|\partial_{t}^{j} \operatorname{div} \psi(t)\right\|_{\left(H^{1}(\Omega(0))\right)^{\prime}} \leq\left\|\partial_{t}^{j} \psi(t)\right\|_{L^{2}(\Omega(0))} .
$$

Integrating in time, we get

$$
\|\operatorname{div} \psi\|_{H^{1+r / 2}\left(0, T ;\left(H^{1}(\Omega(0))\right)^{\prime}\right)} \leq\|\psi\|_{K_{T}^{r+2}(\Omega(0))} .
$$

This inequality extends to arbitrary functions in $K_{T}^{r+2}(\Omega(0))$ which satisfy $\left.\psi\right|_{\Gamma_{0} \cup \partial \mathcal{B}}=0$. It is obvious that for such functions $\operatorname{div} \psi \in L^{2}\left(0, T ; H^{r+1}(\Omega(0))\right)$, and, in summary, the operators

$$
\operatorname{div}: \quad\left\{\psi \in K_{T}^{r+2}(\Omega(0)) /\left.\psi\right|_{\Gamma_{0} \cup \partial \mathcal{B}}=0\right\} \rightarrow L^{2}\left(0, T ; H^{r+1}(\Omega(0))\right)
$$

and

$$
\operatorname{div}: \quad\left\{\psi \in K_{T}^{r+2}(\Omega(0)) /\left.\psi\right|_{\Gamma_{0} \cup \partial \mathcal{B}}=0\right\} \rightarrow H^{1+r / 2}\left(0, T ;\left(H^{1}(\Omega(0))\right)^{\prime}\right)
$$

are linear and continuous. For $r$ not an integer this statement holds by interpolation. Then $\psi \in K_{T}^{r+2}(\Omega(0))$ with $\left.\psi\right|_{\Gamma_{0} \cup \partial \mathcal{B}}=0$ implies $\operatorname{div} \psi \in \hat{K}_{T}^{r}(\Omega(0))$. We have similarly that $\operatorname{div} \psi \in \hat{K}_{T}^{r}(\Omega(0))$ if $\psi \in K_{T}^{r+2}(\Omega(0))$ with $\left.\psi\right|_{\Gamma_{0}}=0$ and $\left.\psi\right|_{\partial \mathcal{B}}=\mathbf{z} \in H^{r / 2+1}\left(0, T ; H^{l}(\partial \mathcal{B})\right)$. Indeed, we consider a lifting $\tilde{\mathbf{z}} \in H^{r / 2+1}\left(0, T ; H^{l+1 / 2}(\Omega(0))\right)$ of z. Then $\operatorname{div} \tilde{\mathbf{z}} \in H^{r / 2+1}\left(0, T ; H^{l-1 / 2}(\Omega(0))\right)$, since $l \geq r+3 / 2$, and thanks to the last argument $\operatorname{div}(\psi-\tilde{\mathbf{z}}) \in$ $\hat{K}_{T}^{r}(\Omega(0))$. Therefore, $\operatorname{div} \psi \in \hat{K}_{T}^{r}(\Omega(0))$ and we have the following estimate

$$
\|\operatorname{div} \psi\|_{H^{1+r / 2}\left(0, T ;\left(H^{1}(\Omega(0))\right)^{\prime}\right)} \leq C\left(\|\psi\|_{K_{T}^{r+2}(\Omega(0))}+\|\mathbf{z}\|_{H^{r / 2+1}\left(0, T ; H^{l}(\partial \mathcal{B})\right)}\right) .
$$

Proposition 2. Suppose that $1<r<3 / 2$. Let $\mathbf{f} \in K_{T}^{r}(\Omega(0)), \mathbf{u}_{0} \in H^{r+1}(\Omega(0)), \rho \in \hat{K}_{T}^{r}(\Omega(0))$ and $\mathbf{v}_{b} \in$ $H^{r / 2+1}\left(0, T ; H^{l}(\partial \mathcal{B})\right)$, with $l \geq r+3 / 2$. We suppose that the compatibility conditions (19) are satisfied, then there exists a unique solution of (18) such that $\mathbf{v} \in K_{T}^{r+2}(\Omega(0)), \nabla p \in K_{T}^{r}(\Omega(0)), \int_{\Omega(0)} p=0$ and

$$
\|\mathbf{v}\|_{K_{T}^{r+2}(\Omega(0))}+\|\nabla p\|_{K_{T}^{r}(\Omega(0))} \leq C(T)\left[\|\mathbf{f}\|_{K_{T}^{r}(\Omega(0))}+\|\rho\|_{\hat{K}_{T}^{r}(\Omega(0))}+\left\|\mathbf{v}_{b}\right\|_{H^{r / 2+1}\left(0, T ; H^{l}(\partial \mathcal{B})\right)}\right]
$$

If, moreover, we make the following additional assumption

$$
\mathbf{f}(0)=0, \quad \rho(0)=\partial_{t} \rho(0)=0, \quad \mathbf{v}_{b}=0, \quad \mathbf{u}_{0}=0,
$$

then the constant $C(T)$ can be chosen independent of $T$. Furthermore, $(\mathbf{v}, p)$ verifies the following conditions, at the time $t=0$ :

$$
\mathbf{v}(0)=\partial_{t} \mathbf{v}(0)=0, \quad p(0)=0 .
$$

Proof of the Proposition 2. We shall prove this proposition by reducing into two steps the problem to the case previously treated, that is to say the homogeneous case. We are going to build continuous lifting of $\mathbf{v}_{b}, \mathbf{u}_{0}$ and $\rho$.

$\circ$ Lifting of $\mathbf{v}_{b}$ and $\mathbf{u}_{0}$.

Thanks to the assertion $i$ ) of Theorem 3, since $r<3 / 2$ and taking into account (19), there exists a function $\phi_{0} \in K_{T}^{r+2}(\Omega(0))$ such that

$$
\left.\phi_{0}\right|_{\partial \mathcal{B}}=\mathbf{v}_{b},\left.\quad \phi_{0}\right|_{\Gamma_{0}}=0, \quad \phi_{0}(0)=\mathbf{u}_{0}, \quad \partial_{t} \phi_{0}(0)=\nu \Delta \mathbf{u}_{0}+\mathbf{f}(0)
$$

and

$$
\left\|\phi_{0}\right\|_{K_{T}^{r+2}(\Omega(0))} \leq C\left[\left\|\mathbf{v}_{b}\right\|_{K_{T}^{r+3 / 2}(\partial \mathcal{B})}+\left\|\mathbf{u}_{0}\right\|_{H^{r+1}(\Omega(0))}+\|\mathbf{f}\|_{K_{T}^{r}(\Omega(0))}\right]
$$


with a constant $C$ which may depend on $T$. Then $\left(\mathbf{v}_{1}=\mathbf{v}-\phi_{0}, p\right)$ satisfies

$$
\begin{cases}\partial_{t} \mathbf{v}_{1}-\nu \Delta \mathbf{v}_{1}+\nabla p=\mathbf{f}-\partial_{t} \phi_{0}+\nu \Delta \phi_{0} \stackrel{\text { def }}{=} \mathbf{f}_{0} & \text { in } \Omega(0), \\ \operatorname{div} \mathbf{v}_{1}=\rho-\operatorname{div} \phi_{0} \stackrel{\text { def }}{=} \sigma_{0} & \text { in } \Omega(0), \\ \mathbf{v}_{1}=0 & \text { on } \partial \mathcal{B} \cup \Gamma_{0}, \\ \mathbf{v}_{1}(0)=0 & \text { in } \Omega(0),\end{cases}
$$

with, thanks to the choice of $\phi_{0}$ and (19)

$$
\mathbf{f}_{0}(0)=0, \sigma_{0}(0)=0
$$

Moreover, recalling the remark we made before the proposition 2, div $\phi_{0}$ and then $\sigma_{0}$ belong to $\hat{K}_{T}^{r}(\Omega(0))$. $\circ$ Lifting of the divergence.

The next step is to adjust the divergence. We want a function $\phi_{1}$ which satisfies:

$$
\phi_{1} \in K_{T}^{r+2}(\Omega(0)), \phi_{1}(0)=0, \operatorname{div} \phi_{1}=\sigma_{0} \text { and }\left.\phi_{1}\right|_{\partial \Omega(0)}=0 .
$$

Lemma 1 implies that there exists an extension $\bar{\sigma}$ of $\sigma_{0}$ such that

$$
\bar{\sigma} \in L^{2}\left(\mathbb{R} ; H^{r+1}(\Omega(0))\right) \cap H^{1+r / 2}\left(\mathbb{R} ;\left(H^{1}(\Omega(0))\right)^{\prime}\right),
$$

and

$$
\|\bar{\sigma}\|_{L^{2}\left(\mathbb{R} ; H^{r+1}(\Omega(0))\right) \cap H^{1+r / 2}\left(\mathbb{R} ;\left(H^{1}(\Omega(0))\right)^{\prime}\right)} \leq C\left\|\sigma_{0}\right\|_{L^{2}\left(0, T ; H^{r+1}(\Omega(0)) \cap H^{1+r / 2}\left(0, T ;\left(H^{1}(\Omega(0))\right)^{\prime}\right)\right.},
$$

with a constant $C$ which may depend on $T$. Since the average of $\sigma_{0}$ is equal to zero, we can choose the average of $\bar{\sigma}$ equal zero (extension by reflexion). Denoting by $\hat{\bar{\sigma}}$ the Fourier transform of $\bar{\sigma}$, we remark that

$$
\hat{\bar{\sigma}} \in L^{2}\left(\mathbb{R} ; H^{r+1}(\Omega(0))\right) \quad \text { and } \quad|\tau|^{1+r / 2} \hat{\bar{\sigma}} \in L^{2}\left(\mathbb{R} ;\left(H^{1}(\Omega(0))\right)^{\prime}\right),
$$

and the average of $\hat{\bar{\sigma}}$ is equal to zero. For each $\tau$ in $\mathbb{R}$, we define $\theta$ by

$$
\left\{\begin{aligned}
-\Delta \theta(\tau) & =\hat{\bar{\sigma}}(\tau) & & \text { in } \Omega(0) \\
\frac{\partial \theta(\tau)}{\partial \mathbf{n}} & =0 & & \text { on } \Gamma_{0} \cup \partial \mathcal{B} .
\end{aligned}\right.
$$

We search the function $\theta$ so that $\int_{\Omega(0)} \theta=0$. For almost all $\tau, \theta(\tau) \in H^{r+3}(\Omega(0))$ and $\|\theta(\tau)\|_{H^{r+3}(\Omega(0))} \leq$ $C\|\hat{\bar{\sigma}}(\tau)\|_{H^{r+1}(\Omega(0))}$ with $C$ independent of $\tau$. Furthermore, from the variational equivalent formulation we deduce

$$
\|\theta(\tau)\|_{H^{1}(\Omega(0))} \leq C\|\hat{\bar{\sigma}}(\tau)\|_{\left(H^{1}(\Omega(0))\right)^{\prime}} .
$$

Hence $\theta \in L^{2}\left(\mathbb{R} ; H^{r+3}(\Omega(0))\right)$ and $|\tau|^{1+r / 2} \nabla \theta \in L^{2}\left(\mathbb{R} ; L^{2}(\Omega(0))\right)$. We set $\psi_{1}=\nabla F^{-1}(\theta)$, where $F^{-1}$ denotes the inverse Fourier transform. Then, the function $\psi_{1}$ belongs to $K_{T}^{r+2}(\Omega(0))$ and satisfies

$$
\psi_{1}(0)=0, \operatorname{div} \psi_{1}=\sigma_{0},\left.\quad \psi_{1} \cdot \mathbf{n}\right|_{\Gamma_{0} \cup \partial \mathcal{B}}=0, \quad \Pi \psi_{1}=0 .
$$

The last equality comes from the fact that $\psi_{1}$ is a gradient. We have just built a function in $K_{T}^{r+2}(\Omega(0))$ whose divergence is equal to $\sigma_{0}$ and so that its normal component on the boundary $\Gamma_{0} \cup \partial \mathcal{B}$ is zero. We shall add to $\psi_{1}$ 
a function $\psi_{2}$ that will not modify the divergence but such as $\psi_{1}+\psi_{2}$ satisfies the desired boundary conditions. We choose $\psi_{2}=\operatorname{curl} w$ with $w$ such that:

$$
\left\{\begin{array}{l}
\text { on } \Gamma_{0} \cup \partial \mathcal{B}: \quad w=0, \frac{\partial w}{\partial \mathbf{n}}=-\psi_{1} \wedge \mathbf{n} \\
\text { at } t=0: \quad w(0)=\partial_{t} w(0)=0 .
\end{array}\right.
$$

The compatibility conditions between the traces to ensure the existence of $w$ in $K_{T}^{r+3}(\Omega(0))$ are satisfied, because $r<3 / 2$ and $\sigma_{0}(0)=0$. We set $\phi_{1}=\psi_{1}+\psi_{2}=\nabla F^{-1}(\theta)+\operatorname{curl} w$. The choice of $w$ implies that the function $\phi_{1} \in K_{T}^{r+2}(\Omega(0))$ and

The pair $\left(\mathbf{v}_{2}=\mathbf{v}_{1}-\phi_{1}, p\right)$ then satisfies:

$$
\phi_{1}(0)=0, \operatorname{div} \phi_{1}=\sigma_{0}, \quad \phi_{1}=0 \text { on } \partial \Omega(0) .
$$

$$
\begin{cases}\partial_{t} \mathbf{v}_{2}-\nu \Delta \mathbf{v}_{2}+\nabla p=\mathbf{f}_{0}-\partial_{t} \phi_{1}+\nu \Delta \phi_{1} \stackrel{\text { def }}{=} \mathbf{f}_{1} & \text { in } \Omega(0), \\ \operatorname{div} \mathbf{v}_{2}=0 & \text { in } \Omega(0), \\ \mathbf{v}_{2}=0 & \text { on } \partial \mathcal{B} \cup \Gamma_{0}, \\ \mathbf{v}_{2}(0)=0 & \text { in } \Omega(0)\end{cases}
$$

Therefore, we are back to the homogeneous case with a right hand side $\mathbf{f}_{1}$ such that $\Pi \mathbf{f}_{1}(0)=0$. Indeed, $\mathbf{f}_{0}(0)=0, \psi_{1}(0)=\psi_{2}(0)=\partial_{t} \psi_{2}(0)=0$ and $\Pi \psi_{1}=0$. Then there exists a unique solution $\left(\mathbf{v}_{2}, p\right)$ of $(27)$ such as $\mathbf{v}_{2} \in K_{T}^{r+2}(\Omega(0)), \nabla p \in K_{T}^{r}(\Omega(0)), \int_{\Omega(0)} p=0$ and

$$
\left\|\mathbf{v}_{2}\right\|_{K_{T}^{r+2}(\Omega(0))}+\|\nabla p\|_{K_{T}^{r}(\Omega(0))} \leq C\left\|\mathbf{f}_{1}\right\|_{K_{T}^{r}(\Omega(0))} .
$$

But

$$
\begin{aligned}
& \left\|\phi_{0}\right\|_{K_{T}^{r+2}(\Omega(0))} \leq C\left[\left\|\mathbf{v}_{b}\right\|_{K_{T}^{r+3 / 2}(\partial \mathcal{B})}+\left\|\mathbf{u}_{0}\right\|_{H^{r+1}(\Omega(0))}+\|\mathbf{f}\|_{K_{T}^{r}(\Omega(0))}\right], \\
& \left\|\phi_{1}\right\|_{K_{T}^{r+2}(\Omega(0))} \leq C\left[\|\rho\|_{\tilde{K}_{T}^{r}(\Omega(0))}+\left\|\phi_{0}\right\|_{K_{T}^{r+2}(\Omega(0))}+\left\|\mathbf{v}_{b}\right\|_{H^{r / 2+1}\left(0, T ; H^{l}(\partial \mathcal{B})\right)}\right],
\end{aligned}
$$

with a constant $C$ which may depend on $T$. Consequently, there exists a unique solution $(\mathbf{v}, p)$ of $(18)$ such that $\mathbf{v} \in K_{T}^{r+2}(\Omega(0)), \nabla p \in K_{T}^{r}(\Omega(0)), \int_{\Omega(0)} p=0$ and

$$
\begin{aligned}
\|\mathbf{v}\|_{K_{T}^{r+2}(\Omega(0))}+\|\nabla p\|_{K_{T}^{r}(\Omega(0))} \leq & C\left[\|\mathbf{f}\|_{K_{T}^{r}(\Omega(0))}+\|\rho\|_{\hat{K}_{T}^{r}(\Omega(0))}\right. \\
& \left.+\left\|\mathbf{v}_{b}\right\|_{H^{r / 2+1}\left(0, T ; H^{l}(\partial \mathcal{B})\right)}+\left\|\mathbf{u}_{0}\right\|_{H^{r+1}(\Omega(0))}\right] .
\end{aligned}
$$

If the condition (23) is satisfied then the constant $C$ can be chosen independent of the time $T$ because at the second step the extension of divergence can be done with a bound which is independent of $T$ since $\rho(0)=\partial_{t} \rho(0)=0$.

Proof of the last statement of Proposition 2. We suppose that (23) is satisfied. By definition of $\mathbf{v}$ we have $\mathbf{v}(0)=0$. Now, we have to prove that $\partial_{t} \mathbf{v}(0)=0$ and $p(0)=0$. We have

$$
\begin{aligned}
\operatorname{div} \partial_{t} \mathbf{v}(0) & =0 \\
\partial_{t} \mathbf{v}(0)+\nabla p(0) & =0 \\
\left.\mathbf{v}\right|_{\Gamma_{0} \cup \partial \mathcal{B}} & =0 .
\end{aligned}
$$

We recall that $\Pi$ denotes the $L^{2}(\Omega(0))$-projection operator on $H$. We have $(I-\Pi) \mathbf{v}=\nabla \chi$, where $\chi$ is defined by

$$
(\nabla \chi, \nabla \phi)=-(\rho, \phi), \quad \forall \phi \in H^{1}(\Omega(0))
$$


Indeed,

$$
((I-\Pi) \mathbf{v}), \nabla \phi)=(\mathbf{v}, \nabla \phi), \quad \forall \phi \in H^{1}(\Omega(0))
$$

The relation (28) implies that $\nabla \partial_{t} \chi(0)=0$ and then that $\partial_{t} \mathbf{v}(0)=\Pi\left(\partial_{t} \mathbf{v}(0)\right)$. But $\Pi \nabla p(0)=0(\nabla p(0) \in$ $\left.L^{2}(\Omega(0))\right)$, therefore projecting the equation (29) on divergence free vector, we obtain $\Pi\left(\partial_{t} \mathbf{v}(0)\right)=0$, that implies that $\partial_{t} \mathbf{v}(0)=0$. Then the pressure at the time $t=0$ is constant but since $\int_{\Omega(0)} p(0)=0$, this constant is zero. This ends the proof of the Proposition 2.

Remark 4. We can rewrite the conclusion of Proposition 2 in terms of operators. If we denote by $L$ the linear operator

$$
(\mathbf{w}, q) \in X_{T}^{r} \mapsto\left(\partial_{t} \mathbf{w}-\nu \Delta \mathbf{w}+\nabla q, \nabla \cdot \mathbf{w},\left.\mathbf{w}\right|_{\partial \mathcal{B}}, \mathbf{w}(0)\right) \in Y_{T}^{r}
$$

with

$$
X_{T}^{r}=\left\{(\mathbf{w}, q), \mathbf{w} \in K_{T}^{r+2}(\Omega(0)), \nabla q \in K_{T}^{r}(\Omega(0)), \int_{\Omega(0)} q=0,\left.\mathbf{w}\right|_{\partial B} \in H^{r / 2+1}\left(0, T ; H^{l}(\partial \mathcal{B})\right),\left.\mathbf{w}\right|_{\Gamma_{0}}=0\right\}
$$

and

$$
Y_{T}^{r}=\left\{\left(\mathbf{f}, \rho, \mathbf{v}_{b}, \mathbf{u}_{0}\right), \mathbf{f} \in K_{T}^{r}(\Omega(0)), \rho \in \hat{K}_{t}^{r}(\Omega(0)), \mathbf{v}_{b} \in H^{r / 2+1}\left(0, T ; H^{l}(\partial \mathcal{B})\right), \mathbf{u}_{0} \in H^{r+1}(\Omega(0))\right\},
$$

then $L$ has a bounded inverse for any $1<r<3 / 2$. Moreover, if we set

$$
X_{T, 0}^{r}=\left\{(\mathbf{w}, q) \in X_{T}^{r} / \mathbf{w}(0)=\partial_{t} \mathbf{w}(0)=0,\left.\mathbf{w}\right|_{\partial \mathcal{B}}=0, q(0)=0\right\},
$$

and

$$
Y_{T, 0}^{r}=\left\{(\mathbf{f}, \rho, 0,0) \in Y_{T}^{r} \quad / \mathbf{f}(0)=0, \rho(0)=\partial_{t} \rho(0)=0\right\},
$$

then $L: X_{T, 0}^{r} \longrightarrow Y_{T, 0}^{r}$ has a bounded inverse with a norm independent of $T$.

\subsection{Estimates of the nonlinear terms. Proof of Theorem 2}

In what follows, we choose an arbritary time $T_{0}$. We suppose that $\mathbf{v}_{b} \in H^{r / 2+1}\left(0, T_{0} ; H^{l}(\partial \mathcal{B})\right)$ and that the condition (13) is satisfied. Let $(\mathbf{v}, p)$ be a solution of $(12)$ then $(\mathbf{v}, p)$ verifies:

$$
\begin{cases}\partial_{t} \mathbf{v}-\nu \Delta \mathbf{v}+\nabla p=\mathbf{f} \circ \chi_{\mathbf{v}}-\nu \Delta \mathbf{v}+\nu\left(\nabla_{\mathbf{v}}\right)^{2} \mathbf{v}+\left(\nabla-\nabla_{\mathbf{v}}\right) p & \text { in } \Omega(0) \\ \operatorname{div} \mathbf{v}=\left(\nabla-\nabla_{\mathbf{v}}\right) \cdot \mathbf{v} & \text { in } \Omega(0) \\ \mathbf{v}=0 & \text { on } \Gamma_{0} \\ \mathbf{v}=\mathbf{v}_{b} & \text { on } \partial \mathcal{B} \\ \mathbf{v}(0)=\mathbf{u}_{0} & \text { in } \Omega(0) .\end{cases}
$$

In a first step, we shall build $\left(\mathbf{v}_{0}, p_{0}\right)$ such that, if we set

$$
(\tilde{\mathbf{v}}, \tilde{p})=\left(\mathbf{v}-\mathbf{v}_{0}, p-p_{0}\right),
$$

then we have

$$
\tilde{\mathbf{v}}(0)=\partial_{t} \tilde{\mathbf{v}}(0)=0, \quad \tilde{p}(0)=0
$$

In order to define $\left(\mathbf{v}_{0}, p_{0}\right)$ let us analyse some necessary conditions to (33). Assuming a solution ( $\left.\mathbf{v}, p\right)$ is known, we differentiate with respect to time the equation $\nabla_{\mathbf{v}} \cdot \mathbf{v}=0$ and set $t=0$. We recall that $\nabla_{\mathbf{v}} \cdot \mathbf{v}=$ $\left(\operatorname{cof}\left(I+\int_{0}^{t} \nabla \mathbf{v}\right) \cdot \nabla\right) \cdot \mathbf{v}$. We have

$$
\nabla . \partial_{t} \mathbf{v}(0)=\left(\left(\nabla \mathbf{u}_{0}\right)^{T} \cdot \nabla\right) \mathbf{u}_{0} \stackrel{\text { def }}{=} \sigma_{1}
$$


because $\mathbf{v}(0)=\mathbf{u}_{0}$. Therefore, if $\left(\mathbf{v}_{0}, p_{0}\right)$ exists it has to satisfy $\nabla . \partial_{t} \mathbf{v}_{0}(0)=\sigma_{1}$. Since $\nabla \mathbf{u}_{0} \in H^{r}(\Omega(0))$, with $r>1$ we have $\sigma_{1} \in H^{r-1}(\Omega(0))$. By Theorem 3, there exists $\sigma_{0} \in K_{T_{0}}^{r+2}(\Omega(0))$ such that $\sigma_{0}(0)=0$ and $\partial_{t} \sigma_{0}(0)=\sigma_{1}$. We consider now $\mathbf{z} \in H^{r / 2+1}\left(0, T_{0} ; H^{l}(\partial \mathcal{B})\right)$ such that

$$
\left\{\begin{aligned}
\mathbf{z}(0) & =\mathbf{v}_{b}(0) \\
\int_{\partial \mathcal{B}}^{\mathbf{z} . \mathbf{n}} & =\int_{\Omega(0)} \sigma_{0} .
\end{aligned}\right.
$$

For instance, we can choose $\mathbf{z}=\left.\mathbf{u}_{0}\right|_{\partial \mathcal{B}}+\alpha(t) \mathbf{w}$ where $\mathbf{w}$ denotes a smooth function which does not depend on the time variable $t$ and so that $\int_{\partial \mathcal{B}} \mathbf{w} \cdot \mathbf{n}=1$ and $\alpha(t)=\int_{\Omega(0)} \sigma_{0}$. Taking into account the regularities of $\sigma_{0}$, $\alpha$ belongs to $H^{r / 2+1}\left(0, T_{0}\right)$. Then $\mathbf{z}$ satisfies (34) and belongs to $H^{r / 2+1}\left(0, T_{0} ; H^{l}(\partial \mathcal{B})\right)$, because we made the assumption that $\left.\mathbf{u}_{0}\right|_{\partial \mathcal{B}}$ is in $H^{l}$. We are now in a position of defining $\left(\overline{\mathbf{v}}_{0}, p_{0}\right) \in X_{T_{0}}^{r}$ solution of (Prop. 2 gives the existence of such a solution):

$$
\begin{cases}\partial_{t} \overline{\mathbf{v}}_{0}-\nu \Delta \overline{\mathbf{v}}_{0}+\nabla p_{0}=\mathbf{f}(0) & \text { in } \Omega(0) \\ \operatorname{div} \overline{\mathbf{v}}_{0}=\sigma_{0} & \text { in } \Omega(0) \\ \overline{\mathbf{v}}_{0}=0 & \text { on } \Gamma_{0} \\ \overline{\mathbf{v}}_{0}=\mathbf{z} & \text { on } \partial \mathcal{B} \\ \overline{\mathbf{v}}_{0}(0)=\mathbf{u}_{0} & \text { in } \Omega(0)\end{cases}
$$

We associate a new velocity $\underline{\mathbf{v}}_{0}$ in $K_{T_{0}}^{r+2}(\Omega(0))$ such that

$$
\underline{\mathbf{v}}_{0}(0)=\partial_{t} \underline{\mathbf{v}}_{0}(0)=0,\left.\quad \underline{\mathbf{v}}_{0}\right|_{\partial \mathcal{B}}=\mathbf{v}_{b}-\mathbf{z},\left.\quad \underline{\mathbf{u}}_{0}\right|_{\Gamma_{0}}=0
$$

We finally set $\left(\mathbf{v}_{0}, p_{0}\right)=\left(\overline{\mathbf{v}}_{0}+\underline{\mathbf{v}}_{0}, p_{0}\right)$. Then $(\tilde{\mathbf{v}}, \tilde{p})$ define in $(32)$ is solution of

$$
\begin{cases}\partial_{t} \tilde{\mathbf{v}}-\nu \Delta \tilde{\mathbf{v}}+\nabla \tilde{p}=\mathbf{f} \circ \chi_{\tilde{\mathbf{v}}+\mathbf{v}_{0}}-\mathbf{f}(0)+\alpha_{1}\left(\mathbf{v}_{0}+\tilde{\mathbf{v}}, p_{0}+\tilde{p}\right)-\partial_{t} \underline{\mathbf{v}}_{0}+\nu \Delta \underline{\mathbf{v}}_{0} & \text { in } \Omega(0), \\ \operatorname{div} \tilde{\mathbf{v}}=\alpha_{2}\left(\mathbf{v}_{0}+\tilde{\mathbf{v}}\right)-\operatorname{div} \mathbf{v}_{0} & \text { in } \Omega(0), \\ \tilde{\mathbf{v}}=0 & \text { on } \Gamma_{0} \cup \partial \mathcal{B}, \\ \tilde{\mathbf{v}}(0)=0 & \text { in } \Omega(0),\end{cases}
$$

with

and

$$
\alpha_{1}(\mathbf{w}, q)=-\nu \Delta \mathbf{w}+\nu\left(\nabla_{\mathbf{w}}\right)^{2} \mathbf{w}+\left(\nabla-\nabla_{\mathbf{w}}\right) q,
$$

We can also write that $(\tilde{\mathbf{v}}, \tilde{p})$ is solution of

$$
\alpha_{2}(\mathbf{w})=\left(\nabla-\nabla_{\mathbf{w}}\right) \cdot \mathbf{w}
$$

$$
L(\tilde{\mathbf{v}}, \tilde{p})=\left(\mathbf{f} \circ \chi_{\tilde{\mathbf{v}}+\mathbf{v}_{0}}-\mathbf{f}(0)+\alpha_{1}\left(\tilde{\mathbf{v}}+\mathbf{v}_{0}, \tilde{p}+p_{0}\right), \alpha_{3}\left(\mathbf{v}_{0}+\tilde{\mathbf{v}}\right), 0,0\right)+\left(-\partial_{t} \underline{\mathbf{v}}_{0}+\nu \Delta \underline{\mathbf{v}}_{0}, \rho_{0}, 0,0\right),
$$

where $\alpha_{3}(\mathbf{w})=\alpha_{2}(\mathbf{w})-\left(\nabla-\nabla_{\mathbf{v}_{0}}\right) \cdot \mathbf{v}_{0}$. We set $\rho_{0}=-\nabla_{\mathbf{v}_{0}} \cdot \mathbf{v}_{0}$. We want to use the results we obtain for the linear problem in order to obtain the solvability of the modified nonlinear fluid problem (36) for a given velocity on the disc. To do so we will use a fixed point theorem. We introduce the mapping

$$
\left(\mathbf{v}_{1}, p_{1}\right) \in X_{T, 0}^{r} \mapsto\left(\mathbf{v}_{2}, p_{2}\right) \in X_{T, 0}^{r},
$$

where $\left(\mathbf{v}_{2}, p_{2}\right)$ is defined by

$$
\begin{aligned}
\left(\mathbf{v}_{2}, p_{2}\right)= & L^{-1}\left(\mathbf{f} \circ \chi_{\mathbf{v}_{1}+\mathbf{v}_{0}}-\mathbf{f}(0)+\alpha_{1}\left(\mathbf{v}_{1}+\mathbf{v}_{0}, p_{1}+p_{0}\right), \alpha_{3}\left(\mathbf{v}_{1}+\mathbf{v}_{0}\right), 0,0\right) \\
& +L^{-1}\left(-\partial_{t} \underline{\mathbf{v}}_{0}+\nu \Delta \underline{\mathbf{v}}_{0}, \rho_{0}, 0,0\right) .
\end{aligned}
$$


We check that this mapping satisfies the contraction mapping principle, for a small enough time. We shall show that the terms on the right hand side (that is to say the nonlinear terms) are defined, that we can control their norms with respect to the time, and that each is lipschitz with a small lipschitz constant. We state now properties on the functions $\alpha_{1}, \alpha_{2} \ldots$ We take two couples $\left(\mathbf{v}_{0}^{i}, p_{0}\right)$ in $X_{T_{0}}^{r}$ with the velocities defined as above: $\mathbf{v}_{0}^{i}=\overline{\mathbf{v}}_{0}+\underline{\mathbf{v}}_{0}^{i}$ on the time interval $\left(0, T_{0}\right)$. These two velocities are associated with two different velocities on the boundary $: \mathbf{v}_{b}^{1}, \mathbf{v}_{b}^{2}$. The functions $\mathbf{v}_{0}^{i}$ satisfy the following assumptions

$$
\begin{aligned}
& \mathbf{v}_{0}^{i}(0)=\mathbf{u}_{0}, \\
& \left.\mathbf{v}_{0}^{i}\right|_{\partial \mathcal{B}}=\mathbf{v}_{b}^{i}, \\
& \mathbf{v}_{0}^{i} \in K_{T_{0}}^{r+2}(\Omega(0)) .
\end{aligned}
$$

Lemma 7. For all $(\mathbf{w}, q) \in X_{T}^{r}, 1<r<3 / 2$, we have $\alpha_{1}(\mathbf{w}, q) \in K_{T}^{r}(\Omega(0))$. Moreover, for a given real number $R>0$ there exists constants $C_{1}, C_{2}$ and $\eta$ depending only on $R, r$ and $\left\|\left(\mathbf{v}_{0}^{i}, p_{0}\right)\right\|_{X_{T_{0}}^{r}}$ such that

$$
\forall T \leq T_{0}, \forall\left(\mathbf{v}_{i}, p_{i}\right) \in X_{T, 0}^{r}, i=1,2 \text { with }\left\|\left(\mathbf{v}_{i}, p_{i}\right)\right\|_{X_{T}^{r}} \leq R
$$

we have

$$
\begin{gathered}
\left\|\alpha_{1}\left(\mathbf{v}_{i}+\mathbf{v}_{0}^{i}, p_{i}+p_{0}\right)\right\|_{K_{T}^{r}(\Omega(0))} \leq C_{1} T^{\eta} \\
\left\|\alpha_{1}\left(\mathbf{v}_{1}+\mathbf{v}_{0}^{1}, p_{1}+p_{0}\right)-\alpha_{1}\left(\mathbf{v}_{2}+\mathbf{v}_{0}^{2}, p_{2}+p_{0}\right)\right\|_{K_{T}^{r}(\Omega(0))} \leq C_{2} T^{\eta}\left\|\left(\mathbf{v}_{1}+\mathbf{v}_{0}^{1}, p_{1}\right)-\left(\mathbf{v}_{2}+\mathbf{v}_{0}^{2}, p_{2}\right)\right\|_{X_{T}^{r}}
\end{gathered}
$$

Proof. Using the Einstein convention for summation, we can make explicit $\alpha_{1}$ in $2 D$ as follows

$$
\begin{aligned}
\alpha_{1}(\mathbf{w}, q)= & \nu \partial_{j}\left(\left(\operatorname{cof}\left(\nabla \int_{0}^{t} \mathbf{w}\right)\right)_{k j}\left[\delta_{k i}+\left(\operatorname{cof}\left(\nabla \int_{0}^{t} \mathbf{w}\right)_{k i}\right] \partial_{i} \mathbf{w}\right)\right. \\
& +\nu \partial_{k}\left(\operatorname{cof}\left(\nabla \int_{0}^{t} \mathbf{w}\right)_{k i} \partial_{i} \mathbf{w}\right)-\operatorname{cof}\left(\nabla \int_{0}^{t} \mathbf{w}\right) \nabla q,
\end{aligned}
$$

where $\delta_{k j}$ denotes the Kronecker symbol. In $3 D$ the complete explicit form is more tedious to write down but we can notice that (as in $2 D$ ) it takes the form

$$
\alpha_{1}(\mathbf{w}, q)=\sum_{j} \partial_{j}\left[\left(P\left(\nabla \int_{0}^{t} \mathbf{w}\right) \cdot \nabla\right) \mathbf{w}\right]+Q\left(\nabla \int_{0}^{t} \mathbf{w}\right) . \nabla q
$$

where $P\left(\nabla \int_{0}^{t} \mathbf{w}\right)$ and $Q\left(\nabla \int_{0}^{t} \mathbf{w}\right)$ are two matrices and each of their component is a polynomial function with respect to the components of $\nabla \int_{0}^{t} \mathbf{w}$ of degree less than 3 and of variance 1 . The $K_{T}^{r}$ norm is composed of two parts the $L^{2}\left(0, T ; H^{r}(\Omega)\right)$ and the $H^{r / 2}\left(0, T ; L^{2}(\Omega)\right)$. Let us first study the $L^{2}\left(0, T ; H^{r}(\Omega)\right)$ part. If $(\mathbf{w}, q) \in X_{T}^{r}$, then $\nabla \mathbf{w} \in K_{T}^{r+1}(\Omega(0))$ and then $\int_{0}^{t} \nabla \mathbf{w} \in H^{1}\left(0, T ; H^{r+1}(\Omega(0))\right) \cap H^{r / 2+3 / 2}\left(0, T ; L^{2}(\Omega(0))\right)$. In particular, $\int_{0}^{t} \nabla \mathbf{w} \in L^{\infty}\left(0, T ; H^{r+1}(\Omega(0))\right)$. Since $\nabla \mathbf{w} \in L^{2}\left(0, T ; H^{r+1}(\Omega(0))\right), \nabla q \in L^{2}\left(0, T ; H^{r}(\Omega(0))\right)$ and $r>1$, Lemma 4 leads to $\alpha_{1}(\mathbf{w}, q) \in L^{2}\left(0, T ; H^{r}(\Omega(0))\right)$. In another hand, we have by interpolation $\int_{0}^{t} \nabla \mathbf{w} \in H^{p+1}\left(0, T ; H^{r+1-2 p}(\Omega(0))\right)$ for $0 \leq p \leq(r+1) / 2$ (see Lem. 2). Therefore, for $p=r / 4$ we have 
$\int_{0}^{t} \nabla \mathbf{w} \in H^{r / 4+1}\left(0, T ; H^{r / 2+1}(\Omega(0))\right)$. But $\nabla \mathbf{w} \in H^{r / 2}\left(0, T ; H^{1}(\Omega(0))\right)$ and $\nabla q \in H^{r / 2}\left(0, T ; L^{2}(\Omega(0))\right)$, then, since $r>1$ and $1+r / 4>r / 2$ we have $\alpha_{1}(\mathbf{w}, q) \in H^{r / 2}\left(0, T ; L^{2}(\Omega(0))\right)$.

Now we shall estimate $\alpha_{1}\left(\mathbf{v}+\mathbf{v}_{0}, p+p_{0}\right)$ for $(\mathbf{v}, p) \in X_{T, 0}^{r}, 1<r<3 / 2$. We do not precise here the exponent $i$.

Thanks to the Cauchy Schwartz inequality, we get

$$
\left\|\int_{0}^{t} \nabla \mathbf{v}_{0}\right\|_{L^{\infty}\left(0, T ; H^{r+1}(\Omega(0))\right)} \leq T^{1 / 2}\left\|\mathbf{v}_{0}\right\|_{K_{T_{0}}^{r+2}(\Omega(0))},
$$

and

$$
\left\|\int_{0}^{t} \nabla \mathbf{v}\right\|_{L^{\infty}\left(0, T ; H^{r+1}(\Omega(0))\right)} \leq T^{1 / 2}\|\mathbf{v}\|_{K_{T}^{r+2}(\Omega(0))} .
$$

Then, recalling the expression of $\alpha_{1}$, there exists some constants $\eta>0$ and $C_{1}$ which depends on $R, r$ and $\left\|\left(\mathbf{v}_{0}, p_{0}\right)\right\|_{X_{T}^{r}}$ such that

$$
\forall(\mathbf{v}, p) \in X_{T, 0}^{r},\|(\mathbf{v}, p)\|_{X_{T}^{r}} \leq R,\left\|\alpha_{1}\left(\mathbf{v}+\mathbf{v}_{0}, p+p_{0}\right)\right\|_{L^{2}\left(0, T ; H^{r}(\Omega(0))\right)} \leq C_{1} T^{\eta}
$$

We study now the second contribution $H^{r / 2}\left(0, T ; L^{2}(\Omega(0))\right)$ of the $K_{T}^{r}$ norm.

$$
\left\|\nabla \mathbf{v}_{0}\right\|_{H^{r / 2}\left(0, T ; H^{1}(\Omega(0))\right)} \leq C\left(T_{0}\right)\left\|\mathbf{v}_{0}\right\|_{K_{T_{0}}^{r+2}(\Omega(0))}
$$

and applying Lemma $2 \mathrm{ii}$ ),

$$
\|\nabla \mathbf{v}\|_{H^{r / 2}\left(0, T ; H^{1}(\Omega(0))\right)} \leq C\|\mathbf{v}\|_{K_{T}^{r+2}(\Omega(0))},
$$

where $C$ denotes a constant independent of $T$ because the velocity $\mathbf{v}$ satisfies $\mathbf{v}(0)=0$. Furthermore, for $T \leq T_{0}$, using Lemma 2 and Lemma 3 with $s=p$, we obtain for $\varepsilon<p<1 / 2$ and $p \leq(r+1) / 2$,

$$
\left\|\int_{0}^{t} \nabla \mathbf{v}_{0}\right\|_{H^{p+1-\varepsilon}\left(0, T ; H^{r+1-2 p}(\Omega(0))\right)} \leq C T^{\varepsilon}\left\|\mathbf{v}_{0}\right\|_{K_{T_{0}}^{r+2}(\Omega(0))} .
$$

We have the same estimate for $\mathbf{v}$ with a norm $\|\mathbf{v}\|_{K_{T}^{r+2}(\Omega(0))}$ in the right hand side of the inequality and with a constant $C$ that does not depend on $T$ because $\mathbf{v}$ verifies $\mathbf{v}(0)=\partial_{t} \mathbf{v}(0)=0$. We choose $p=r / 4$ and $\varepsilon=r / 8$. Then

$$
\left\|\int_{0}^{t} \nabla \mathbf{v}_{0}\right\|_{H^{1+r / 8}\left(0, T ; H^{1+r / 2}(\Omega(0))\right)} \leq C T^{r / 8}\left\|\mathbf{v}_{0}\right\|_{K_{T_{0}}^{r+2}(\Omega(0))},
$$

and

$$
\left\|\int_{0}^{t} \nabla \mathbf{v}\right\|_{H^{1+r / 8}\left(0, T ; H^{1+r / 2}(\Omega(0))\right)} \leq C T^{r / 8}\|\mathbf{v}\|_{K_{T}^{r+2}(\Omega(0))} .
$$

Using Lemma $5 \mathrm{ii}$ ) we obtain estimates of the terms where $\nabla \mathbf{v}_{0}$ does not appear. For instance, the terms $\left(\int_{0}^{t} \nabla \mathbf{v}_{0}\right)_{k i} \partial_{j} \mathbf{v}$ or $\left(\int_{0}^{t} \nabla \mathbf{v}\right)_{k i} \partial_{j} \mathbf{v}$ are bounded in $H^{r / 2}\left(0, T ; H^{1}(\Omega(0))\right)$ by $C T^{r / 8}$. For the products in which $\nabla \mathbf{v}_{0}$ appears, we cannot apply directly Lemma $\left.5 i i\right)$ since $\mathbf{v}_{0}(0)=\mathbf{u}_{0} \neq 0$. Nevertheless, we can write $\mathbf{v}_{0}=\left(\mathbf{v}_{0}-\mathbf{u}_{0}\right)+\mathbf{u}_{0}$. Then we use Lemma $\left.5 i i\right)$ for the terms $\left(\int_{0}^{t} \nabla \mathbf{v}\right)_{k i} \partial_{j}\left(\mathbf{v}_{0}-\mathbf{u}_{0}\right)$ and remark that, for the 
remaining terms, $\mathbf{u}_{0}$ is a data and does not depend on time. Then there exists a strictly positive constant, we will denote by $\eta$ and strictly positive constant $C_{2}$ which can be chosen independent of $T$ such that

$$
\left\|\alpha_{1}\left(\mathbf{v}+\mathbf{v}_{0}, p+p_{0}\right)\right\|_{H^{r / 2}\left(0, T ; L^{2}(\Omega(0))\right)} \leq C_{2} T^{\eta}
$$

The estimates (39) and (42) lead to the estimate (37). We also check, using the same type of arguments that $\alpha_{1}$ is lipschitz (estimate (38)) .

We now state the same type of lemma for $\alpha_{3}$.

Lemma 8. For all $\mathbf{w} \in K_{T}^{r+2}(\Omega(0))$, with $\left.\mathbf{w}\right|_{\Gamma_{0}}=0$, w $\left.\right|_{\partial \mathcal{B}} \in H^{r / 2+1}\left(0, T ; H^{l}(\partial \mathcal{B})\right), 1<r<3 / 2$, we have $\alpha_{2}(\mathbf{w})\left(\right.$ resp. $\left.\alpha_{3}\right) \in \hat{K}_{T}^{r}(\Omega(0))$. Moreover, for a given $R>0$, for $\mathbf{v}_{0}^{i}$ satisfying the hypothesis $(H)$ there exists $C_{1}, C_{2}$ and $\eta$ depending only on $R, r,\left\|\mathbf{v}_{0}^{i}\right\|_{K_{T_{0}}^{r+2}(\Omega(0))}$ and $\left\|\mathbf{v}_{0}^{i}\right\|_{H^{r / 2+1}\left(0, T_{0} ; H^{l}(\partial \mathcal{B})\right)}$ such that $\forall T \leq T_{0}, \forall\left(\mathbf{v}_{i}, p_{i}\right) \in$ $X_{T, 0}^{r}, i=1,2$ with $\left\|\left(\mathbf{v}_{i}, p_{i}\right)\right\|_{X_{T}^{r}} \leq R$, we have

$$
\begin{gathered}
\left\|\alpha_{3}\left(\mathbf{v}_{i}+\mathbf{v}_{0}^{i}\right)\right\|_{\hat{K}_{T}^{r}(\Omega(0))} \leq C_{1} T^{\eta} \\
\left\|\alpha_{2}\left(\mathbf{v}_{1}+\mathbf{v}_{0}^{1}\right)-\alpha_{2}\left(\mathbf{v}_{2}+\mathbf{v}_{0}^{2}\right)\right\|_{\hat{K}_{T}^{r}(\Omega(0))} \leq \quad \\
\quad C_{2} T^{\eta}\left[\left\|\left(\mathbf{v}_{1}+\mathbf{v}_{0}^{1}\right)-\left(\mathbf{v}_{2}+\mathbf{v}_{0}^{2}\right)\right\|_{K_{T}^{r+2}(\Omega(0))}\right. \\
\\
\left.+\left\|\mathbf{v}_{b}^{1}-\mathbf{v}_{b}^{2}\right\|_{H^{r / 2+1}\left(0, T ; H^{l}(\partial \mathcal{B})\right)}\right] .
\end{gathered}
$$

Proof. We first note that $\alpha_{2}(\mathbf{w})$ is a sum of terms that can be written $\left(P_{d-1}\left(\int_{0}^{t} \nabla \mathbf{w}\right) \cdot \nabla\right) \cdot \mathbf{w}$, where $P_{d-1}\left(\int_{0}^{t} \nabla \mathbf{w}\right)$ is a matrix whose component are polynomial functions with respect to the components of $\int_{0}^{t} \nabla \mathbf{w}$ of degree $d-1$ and variance 1 . We recall the definition of $\hat{K}_{T}^{r}(\Omega(0))$ :

$$
\hat{K}_{T}^{r}(\Omega(0))=L^{2}\left(0, T ; H^{r+1}(\Omega(0))\right) \cap H^{r / 2+1}\left(0, T ;\left(H^{1}(\Omega(0))\right)^{\prime}\right) .
$$

As in Lemma 7 we have $\alpha_{2}(\mathbf{w}) \in L^{2}\left(0, T ; H^{r+1}(\Omega(0))\right)$. The earlier estimates for $\alpha_{1}$ in $L^{2}\left(0, T ; H^{r+1}(\Omega(0))\right)$ apply here for $\alpha_{2}$ (resp. $\left.\alpha_{3}\right)$. We only need to be concern with the estimates in $H^{r / 2+1}\left(0, T ;\left(H^{1}(\Omega(0))\right)^{\prime}\right)$. First if $\mathbf{w} \in K_{T}^{r+2}(\Omega(0))$ then $\left(\int_{0}^{t} \nabla \mathbf{w}\right)_{i j} \partial_{k} \mathbf{w} \in H^{r / 2+1}\left(0, T ;\left(H^{1}(\Omega(0))\right)^{\prime}\right)$. Indeed we have $\nabla \mathbf{w} \in H^{1}\left(0, T ; L^{2}(\Omega(0))\right)$ and $\int_{0}^{t} \nabla \mathbf{w} \in H^{1}\left(0, T ; H^{r+1}(\Omega(0))\right)$. Then,

$$
\alpha_{2}(\mathbf{w}) \in H^{1}\left(0, T ; L^{2}(\Omega(0))\right) \subset H^{1}\left(0, T ;\left(H^{1}(\Omega(0))\right)^{\prime}\right) .
$$

It remains to prove that $\partial_{t} \alpha_{2}(\mathbf{w}) \in H^{r / 2}\left(0, T ;\left(H^{1}(\Omega(0))\right)^{\prime}\right)$. It is straightforward to note that it can be written as the sum of terms of the following four types

- $\left(\int_{0}^{t} \nabla \mathbf{w}\right)_{i j}\left(\partial_{t} \nabla \mathbf{w}\right)_{k l}$,

- $(\nabla \mathbf{w})_{i j}(\nabla \mathbf{w})_{k l}$,

- $\left(\int_{0}^{t} \nabla \mathbf{w}\right)_{i j}\left(\int_{0}^{t} \nabla \mathbf{w}\right)_{m n}\left(\partial_{t} \nabla \mathbf{w}\right)_{k l}$,

- $\left(\int_{0}^{t} \nabla \mathbf{w}\right)_{i j}(\nabla \mathbf{w})_{k l}(\nabla \mathbf{w})_{m n}$. 
Let us start with the two first one. By the discussion preceding Proposition 2, we have $\partial_{t} \nabla \mathbf{w} \in H^{r / 2}\left(0, T ;\left(H^{1}(\Omega(0))\right)^{\prime}\right)$. We know that $\int_{0}^{t} \nabla \mathbf{w} \in H^{1+r / 4}\left(0, T ; H^{1+r / 2}(\Omega(0))\right)$ and recall that $H^{1+r / 2}(\Omega(0))$ is a multiplier of $H^{1}(\Omega(0))$ and $H^{1+r / 4}(0, T)$ is a multiplier of $H^{r / 2}(0, T)$. Then Lemma 5 implies that

$$
\left(\int_{0}^{t} \nabla \mathbf{w}\right)_{i j}\left(\partial_{t} \nabla \mathbf{w}\right)_{k l} \in H^{r / 2}\left(0, T ;\left(H^{1}(\Omega(0))\right)^{\prime}\right) .
$$

We study now the second term $(\nabla \mathbf{w})_{i j}(\nabla \mathbf{w})_{k l}$. We have $\nabla \mathbf{w} \in H^{r / 2}\left(0, T ; H^{1}(\Omega(0))\right)$. The multiplication of a function in $H^{1}(\Omega(0))$ by a function in $H^{1}(\Omega(0))$ belongs to $\left(H^{1}(\Omega(0))\right)^{\prime}$. Together with the fact that $H^{r / 2}(0, T)$ is an algebra we obtain from Lemma 5

$$
(\nabla \mathbf{w})_{i j}(\nabla \mathbf{w})_{k l} \in H^{r / 2}\left(0, T ;\left(H^{1}(\Omega(0))\right)^{\prime}\right) .
$$

And then we get $\partial_{t}\left(\alpha_{2}(\mathbf{w})\right) \in H^{r / 2}\left(0, T ;\left(H^{1}(\Omega(0))\right)^{\prime}\right)$ (resp. for $\left.\alpha_{3}\right)$.

Next we estimate $\alpha_{3}$ in $H^{1+r / 2}\left(0, T ;\left(H^{1}(\Omega(0))\right)^{\prime}\right)$.

As previously $\alpha_{3}\left(\mathbf{v}+\mathbf{v}_{0}, p+p_{0}\right)$ can be written as a sum of six generic terms and the basic ones are

$$
\left(\int_{0}^{t} \nabla \mathbf{v}\right)_{i j}(\nabla \mathbf{v})_{k l},\left(\int_{0}^{t} \nabla \mathbf{v}\right)_{i j}\left(\nabla \mathbf{v}_{0}\right)_{k l},\left(\int_{0}^{t} \nabla \mathbf{v}_{0}\right)_{i j}(\nabla \mathbf{v})_{k l} .
$$

We consider the term $\left(\int_{0}^{t} \nabla \mathbf{v}\right)_{i j}\left(\nabla \mathbf{v}_{0}\right)_{k l}$.

We have

$$
\left\|\nabla \mathbf{v}_{0}\right\|_{H^{1}\left(0, T ; L^{2}(\Omega(0))\right)} \leq C\left\|\nabla \mathbf{v}_{0}\right\|_{K_{T_{0}}^{r+2}(\Omega(0))}
$$

recalling (41) we derive

$$
\left\|\left(\int_{0}^{t} \nabla \mathbf{v}\right)_{i j}\left(\nabla \mathbf{v}_{0}\right)_{k l}\right\|_{H^{1}\left(0, T ; L^{2}(\Omega(0))\right)} \leq C T^{r / 8}
$$

and then

$$
\left\|\alpha_{3}\left(\mathbf{v}+\mathbf{v}_{0}\right)\right\|_{H^{1}\left(0, T ; L^{2}(\Omega(0))\right)} \leq C T^{r / 8}
$$

since the other terms can be handled in the same way (each constant can be chosen independent of $T$ since $\mathbf{v}(0)=$ $\left.\partial_{t} \mathbf{v}(0)=0\right)$. We estimate now $\partial_{t} \alpha_{3}$ in the $H^{r / 2}\left(0, T ;\left(H^{1}(\Omega(0))\right)^{\prime}\right)$ norm and consider $\partial_{t}\left(\left(\int_{0}^{t} \nabla \mathbf{v}\right)_{i j}\left(\nabla \mathbf{v}_{0}\right)_{k l}\right)$ as a generic example. We have again two types of term to study: $\left(\int_{0}^{t} \nabla \mathbf{v}\right)_{i j}\left(\nabla \partial_{t} \mathbf{v}_{0}\right)_{k l}$ and $(\nabla \mathbf{v})_{i j}\left(\nabla \mathbf{v}_{0}\right)_{k l}$.

First we consider $\left(\int_{0}^{t} \nabla \mathbf{v}\right)_{i j}\left(\nabla \partial_{t} \mathbf{v}_{0}\right)_{k l}$.

We have that $\nabla \partial_{t} \mathbf{v}_{0}$ is bounded in $H^{r / 2}\left(0, T ;\left(H^{1}(\Omega(0))\right)^{\prime}\right)$ independently of $T$. The estimate (41), Lemma 4 ii), Lemma 5 ii) (we write $\left.\partial_{t} \mathbf{v}_{0}=\left(\partial_{t} \mathbf{v}_{0}-\partial_{t} \mathbf{v}_{0}(0)\right)+\partial_{t} \mathbf{v}_{0}(0)\right)$ lead to:

$$
\left\|\left(\int_{0}^{t} \nabla \mathbf{v}\right)_{i j}\left(\nabla \partial_{t} \mathbf{v}_{0}\right)_{k l}\right\|_{H^{r / 2}\left(0, T ;\left(H^{1}(\Omega(0))\right)^{\prime}\right)} \leq C T^{r / 8},
$$


where $C$ is independent of $T$. We deduce from Lemma 1 that the condition $\partial_{t} \mathbf{v}(0)=0$ provides a bound of $\nabla \mathbf{v}$ in $H^{r / 2+1}\left(0, T ;\left(H^{1}(\Omega(0))\right)^{\prime}\right)$ independent of $T$, so the same estimates hold for $\left(\int_{0}^{t} \nabla \mathbf{v}\right)_{i j}\left(\nabla \partial_{t} \mathbf{v}\right)_{k l}$ and $\left(\int_{0}^{t} \nabla \mathbf{v}_{0}\right)_{i j}\left(\nabla \partial_{t} \mathbf{v}\right)_{k l}$.

Finally we study $(\nabla \mathbf{v})_{i j}\left(\nabla \mathbf{v}_{0}\right)_{k l}$.

We know on one hand that $\nabla \mathbf{v}_{0}$ is bounded in $H^{r / 2}\left(0, T_{0} ; H^{1}(\Omega(0))\right)$. On the other hand since $\mathbf{v}(0)=0$, we obtain $\nabla \mathbf{v}=\int_{0}^{t} \nabla \partial_{t} \mathbf{v}$ and estimate (15) with $X=L^{2}(\Omega(0)), s=0, \varepsilon=1-r / 2$ implies:

$$
\|\nabla \mathbf{v}\|_{H^{r / 2}\left(0, T ; L^{2}(\Omega(0))\right)} \leq C T^{1-r / 2}\|\nabla \mathbf{v}\|_{H^{1}\left(0, T ; L^{2}(\Omega(0))\right)} \leq C T^{1-r / 2}\|\mathbf{v}\|_{K_{T}^{r+2}(\Omega(0))} .
$$

Then we get

$$
\left\|(\nabla \mathbf{v})_{i j}\left(\nabla \mathbf{v}_{0}\right)_{k l}\right\|_{H^{r / 2}\left(0, T ;\left(H^{1}(\Omega(0))\right)^{\prime}\right)} \leq C T^{1-r / 2} .
$$

We have the same type of estimates for $(\nabla \mathbf{v})_{i j}(\nabla \mathbf{v})_{k l}$ and $\left(\nabla \mathbf{v}_{0}\right)_{i j}(\nabla \mathbf{v})_{k l}$.

Then the estimate (43) is satisfied. The same kind of argument enable us to obtain (44) on $\alpha_{2}$. Indeed, for instance in $2 D$ we can write:

$$
\begin{aligned}
\alpha_{2}\left(\mathbf{v}_{0}^{1}+\mathbf{v}_{1}\right)-\alpha_{2}\left(\mathbf{v}_{0}^{2}+\mathbf{v}_{2}\right)= & \operatorname{cof} \int_{0}^{t} \nabla\left(\mathbf{v}_{0}^{1}+\mathbf{v}_{1}\right): \nabla\left(\mathbf{v}_{0}^{1}+\mathbf{v}_{1}\right)-\operatorname{cof} \int_{0}^{t} \nabla\left(\mathbf{v}_{0}^{2}+\mathbf{v}_{2}\right): \nabla\left(\mathbf{v}_{0}^{2}+\mathbf{v}_{2}\right) \\
= & \operatorname{cof} \int_{0}^{t}\left(\nabla\left(\underline{\mathbf{v}}_{0}^{1}+\mathbf{v}_{1}\right)-\nabla\left(\underline{\mathbf{v}}_{0}^{2}+\mathbf{v}_{2}\right)\right): \nabla\left(\mathbf{v}_{0}^{1}+\mathbf{v}_{1}\right) \\
& +\operatorname{cof} \int_{0}^{t} \nabla\left(\mathbf{v}_{0}^{2}+\mathbf{v}_{2}\right): \nabla\left(\left(\underline{\mathbf{v}}_{0}^{2}+\mathbf{v}_{2}\right)-\underline{\mathbf{v}}_{0}^{1}+\mathbf{v}_{1}\right)
\end{aligned}
$$

We remark that we have two type of terms: cof $\int_{0}^{t} \nabla \Phi_{1}: \nabla \Phi_{2}$ with for $i=1$ or for $i=2: \partial_{t} \Phi_{i}(0)=\Phi_{i}(0)=0$. We follow the same ideas as for estimate (43), noticing moreover that

$$
\begin{aligned}
\left\|\nabla\left(\left(\underline{\mathbf{v}}_{0}^{2}+\mathbf{v}_{2}\right)-\underline{\mathbf{v}}_{0}^{1}+\mathbf{v}_{1}\right)\right\|_{H^{r / 2+1}\left(0, T ;\left(H^{1}(\Omega(0))\right)^{\prime}\right)} \leq & \left\|\left(\mathbf{v}_{1}+\mathbf{v}_{0}^{1}\right)-\left(\mathbf{v}_{2}+\underline{\mathbf{v}}_{0}^{2}\right)\right\|_{K_{T}^{r+2}(\Omega(0))} \\
& +\left\|\mathbf{v}_{b}^{1}-\mathbf{v}_{b}^{2}\right\|_{H^{r / 2+1}\left(0, T ; H^{l}(\partial \mathcal{B})\right)}
\end{aligned}
$$

This ends the proof of Lemma 8.

It remains to study $\mathbf{f} \circ \chi_{\mathbf{w}}-\mathbf{f}(0)$. We suppose that $\mathbf{f} \in C^{\infty}\left([0, T] \times \mathbb{R}^{d}\right)$.

Remark 5. Our interest is not to have the minimal regularity on the force $\mathbf{f}$.

Lemma 9. For all $\mathbf{v} \in K_{T}^{r+2}(\Omega(0))$, we have $\mathbf{f} \circ \chi_{\mathbf{v}} \in K_{T}^{r}(\Omega(0))$. Moreover, for $R>0$ there exists $C$ and $\eta$ strictly positive constants which only depend on $R, r$ and $\left\|\mathbf{v}_{0}^{i}\right\|_{K_{T_{0}}^{r+2}(\Omega(0))}$ such that $\forall T \leq T_{0}, \forall\left(\mathbf{v}_{i}, p_{i}\right) \in X_{T, 0}^{r}, i=$ 1,2 such that $\left\|\left(\mathbf{v}_{i}, p_{i}\right)\right\|_{X_{T}^{r}} \leq R$, we have

$$
\left\|\mathbf{f} \circ \chi_{\mathbf{v}_{i}+\mathbf{v}_{0}^{i}}-\mathbf{f}(0)\right\|_{K_{T}^{r}(\Omega(0))} \leq C T^{\eta}
$$

and

$$
\left\|\mathbf{f} \circ \chi_{\mathbf{v}_{1}+\mathbf{v}_{0}^{1}}-\mathbf{f} \circ \chi_{\mathbf{v}_{2}+\mathbf{v}_{0}^{2}}\right\|_{K_{T}^{r}(\Omega(0))} \leq C T^{\eta}\left\|\mathbf{v}_{1}+\mathbf{v}_{0}^{1}-\left(\mathbf{v}_{2}+\mathbf{v}_{0}^{2}\right)\right\|_{K_{T}^{r+2}(\Omega(0))} .
$$


Proof. First we prove that $\mathbf{f} \circ \chi_{\mathbf{v}} \in K_{T}^{r}(\Omega(0))$ for all $\mathbf{v} \in K_{T}^{r+2}(\Omega(0))$.

Recalling that the regularity of $\chi_{\mathbf{v}}$ is the regularity of $\int_{0}^{t} \mathbf{v}$, we know that $\chi_{\mathbf{v}} \in H^{p+1}\left(0, T ; H^{r+2-2 p}(\Omega(0))\right)$ with $0 \leq p \leq \frac{r+2}{2}$.

First, we choose $p=\frac{r-1}{4}$, then $\chi_{\mathbf{v}} \in H^{3 / 4+r / 4}\left(0, T ; H^{r / 2+5 / 2}(\Omega(0))\right)$. And thus using the Sobolev injections $\chi_{\mathbf{v}} \in C^{0}\left([0, T] ; C^{2}(\bar{\Omega}(0))\right)$. Consequently,

$$
\mathbf{f} \circ \chi_{\mathbf{v}} \in C^{0}\left([0, T] ; C^{2}(\bar{\Omega}(0))\right) \subset L^{2}\left(0, T ; H^{r}(\Omega(0))\right),
$$

because $r<3 / 2$.

Then taking $p=\frac{r+3}{4}$, we obtain $\chi_{\mathbf{v}} \in H^{7 / 4+r / 4}\left(0, T ; H^{r / 2+1 / 2}(\Omega(0))\right)$. But $r>1$, then $\chi_{\mathbf{v}} \in C^{1}\left([0, T] ; C^{0}(\bar{\Omega}(0))\right)$. Therefore,

Finally $\mathbf{f} \circ \chi_{\mathbf{v}} \in K_{T}^{r}(\Omega(0))$.

$$
\mathbf{f} \circ \chi_{\mathbf{v}} \in C^{1}\left([0, T] ; C^{0}(\bar{\Omega}(0))\right) \subset H^{r / 2}\left(0, T ; L^{2}(\Omega(0))\right) .
$$

Next we estimate $\left\|\mathbf{f} \circ \chi_{\mathbf{v}_{0}+\mathbf{v}}-\mathbf{f}(0)\right\|_{K_{T}^{r}(\Omega(0))}$ for all $\mathbf{v} \in K_{T}^{r+2}(\Omega(0))$ which satisfies $\mathbf{v}(0)=\partial_{t} \mathbf{v}(0)=0$ and $\left.\mathbf{v}\right|_{\partial \Omega(0)}=0$.

We set

$$
\mathbf{g}=\mathbf{f} \circ \chi_{\mathbf{v}_{0}+\mathbf{v}}-\mathbf{f}(0) .
$$

We check that $\mathbf{g}(0)=0$ and deduce that $\mathbf{g}=\int_{0}^{t} \partial_{t} \mathbf{g}$. This yields

$$
\|\mathbf{g}\|_{L^{2}\left(0, T ; L^{2}(\Omega(0))\right)} \leq T\left\|\partial_{t} \mathbf{g}\right\|_{L^{2}\left(0, T ; L^{2}(\Omega(0))\right)},
$$

and then

$$
\|\mathbf{g}\|_{H^{1-\varepsilon}\left(0, T ; L^{2}(\Omega(0))\right)} \leq T^{\varepsilon}\|\mathbf{g}\|_{H^{1}\left(0, T ; L^{2}(\Omega(0))\right)} .
$$

We choose $1-\varepsilon=r / 2$, it gives

$$
\|\mathbf{g}\|_{H^{r / 2}\left(0, T ; L^{2}(\Omega(0))\right)} \leq T^{1-r / 2}\|\mathbf{g}\|_{H^{1}\left(0, T ; L^{2}(\Omega(0))\right)} .
$$

But

$$
\|\mathbf{g}\|_{H^{1}\left(0, T ; L^{2}(\Omega(0))\right)}^{2}=\|\mathbf{g}\|_{L^{2}\left(0, T ; L^{2}(\Omega(0))\right)}^{2}+\left\|\partial_{t} \mathbf{g}\right\|_{L^{2}\left(0, T ; L^{2}(\Omega(0))\right)}^{2} .
$$

Under the assumption that $\mathbf{f}$ is $C^{\infty}$, we obtain that for any $T, T \leq T_{0}$,

$$
\|\mathbf{g}\|_{L^{2}\left(0, T ; L^{2}(\Omega(0))\right)} \leq C\|\mathbf{f}\|_{L^{\infty}} .
$$

Furthermore,

$$
\begin{aligned}
\partial_{t} \mathbf{g}(t, \xi) & =\left(\partial_{t} \mathbf{f}\right)\left(t, \chi_{\mathbf{v}+\mathbf{v}_{0}}(t, \xi)\right)+\partial_{t} \chi_{\mathbf{v}+\mathbf{v}_{0}}(t, \xi)(\nabla \mathbf{f})\left(t, \chi_{\mathbf{v}+\mathbf{v}_{0}}(t, \xi)\right) \\
& =\left(\partial_{t} \mathbf{f}\right)\left(t, \chi_{\mathbf{v}+\mathbf{v}_{0}}(t, \xi)\right)+\left(\mathbf{v}+\mathbf{v}_{0}\right)(t, \xi)(\nabla \mathbf{f})\left(t, \chi_{\mathbf{v}+\mathbf{v}_{0}}(t, \xi)\right)
\end{aligned}
$$

We conclude that for all $T \leq T_{0}$

$$
\left\|\partial_{t} \mathbf{g}\right\|_{L^{2}\left(0, T ; L^{2}(\Omega(0))\right)} \leq C
$$

with a constant $C$ depending on $\mathbf{f}, R$ and $\mathbf{v}_{0}$. In summary, we have

$$
\left\|\mathbf{f} \circ \chi_{\mathbf{v}+\mathbf{v}_{0}}-\mathbf{f}(0)\right\|_{H^{1}\left(0, T ; L^{2}(\Omega(0))\right)} \leq C T^{1-r / 2} .
$$


Next we study $\|\mathbf{g}\|_{L^{2}\left(0, T ; H^{r}(\Omega(0))\right)}$. We have already estimated $\mathbf{g}$ in $L^{2}\left(0, T ; L^{2}(\Omega(0))\right)$. In order to simplify the presentation we denote by $\partial_{x}$ all the first order derivatives with respect the spatial variables.

$$
\begin{aligned}
\partial_{x}\left(\mathbf{f} \circ \chi_{\mathbf{v}+\mathbf{v}_{0}}\right)-\partial_{x} \mathbf{f}(0) & =\left(\partial_{x} \mathbf{f}\right) \circ \chi_{\mathbf{v}+\mathbf{v}_{0}} \partial_{x} \chi_{\mathbf{v}+\mathbf{v}_{0}}-\partial_{x} \mathbf{f}(0) \\
& =\left(\partial_{x} \mathbf{f}\right) \circ \chi_{\mathbf{v}+\mathbf{v}_{0}}-\partial_{x} \mathbf{f}(0)+\left(\int_{0}^{t} \partial_{x}\left(\mathbf{v}+\mathbf{v}_{0}\right)\right)\left(\partial_{x} \mathbf{f}\right) \circ \chi_{\mathbf{v}+\mathbf{v}_{0}} .
\end{aligned}
$$

We notice that $\left(\partial_{x} \mathbf{f}\right) \circ \chi_{\mathbf{v}+\mathbf{v}_{0}}(0)=\partial_{x} \mathbf{f}(0)$, we have

$$
\left\|\left(\partial_{x} \mathbf{f}\right) \circ \chi_{\mathbf{v}+\mathbf{v}_{0}}-\partial_{x} \mathbf{f}(0)\right\|_{L^{2}\left(0, T ; L^{2}(\Omega(0))\right)} \leq T\left\|\left(\partial_{x} \mathbf{f}\right) \circ \chi_{\mathbf{v}+\mathbf{v}_{0}}-\partial_{x} \mathbf{f}(0)\right\|_{H^{1}\left(0, T ; L^{2}(\Omega(0))\right)} .
$$

And thus the same study we did previously but with $\partial_{x} \mathbf{f}$ instead of $\mathbf{f}$ leads to

$$
\left\|\left(\partial_{x} \mathbf{f}\right) \circ \chi_{\mathbf{v}+\mathbf{v}_{0}}-\partial_{x} \mathbf{f}(0)\right\|_{L^{2}\left(0, T ; L^{2}(\Omega(0))\right)} \leq C T,
$$

where $C$ denotes a constant depending only on $\mathbf{f}, R$ and $\mathbf{v}_{0}$. We have in a same way,

$$
\begin{aligned}
\left\|\left(\int_{0}^{t} \partial_{x}\left(\mathbf{v}+\mathbf{v}_{0}\right)\right)\left(\partial_{x} \mathbf{f}\right) \circ \chi_{\mathbf{v}+\mathbf{v}_{0}}\right\|_{L^{2}\left(0, T ; L^{2}(\Omega(0))\right)} & \leq C\left\|\int_{0}^{t} \partial_{x}\left(\mathbf{v}+\mathbf{v}_{0}\right)\right\|_{L^{2}\left(0, T ; L^{2}(\Omega(0))\right)} \\
& \leq C T\left\|\partial_{x}\left(\mathbf{v}+\mathbf{v}_{0}\right)\right\|_{L^{2}\left(0, T ; L^{2}(\Omega(0))\right)}
\end{aligned}
$$

Using Lemma 2, we get

$$
\left\|\left(\int_{0}^{t} \partial_{x}\left(\mathbf{v}+\mathbf{v}_{0}\right)\right)\left(\partial_{x} \mathbf{f}\right) \circ \chi_{\mathbf{v}+\mathbf{v}_{0}}\right\|_{L^{2}\left(0, T ; L^{2}(\Omega(0))\right)} \leq C T
$$

with $C$ independent of $T$. In summary

$$
\left\|\partial_{x}\left(\mathbf{f} \circ \chi_{\mathbf{v}+\mathbf{v}_{0}}\right)-\partial_{x} \mathbf{f}(0)\right\|_{L^{2}\left(0, T ; L^{2}(\Omega(0))\right)} \leq C T .
$$

We can obtain the same kind of estimates for the second order derivatives. The inequality (49) is satisfied since $r<3 / 2$. The estimate (50) can be obtain using a Taylor formula applied to $\mathbf{f}$ which is by assumption $C^{\infty}$, and using also Lemma 2 and 3 as we did previously.

We now consider the mapping $S_{1}$ as follows

$$
\left(\mathbf{w}_{1}, q_{1}\right) \in X_{T, 0}^{r} \mapsto\left(\mathbf{w}_{2}, q_{2}\right) \in X_{T, 0}^{r},
$$

where $\left(\mathbf{w}_{2}, q_{2}\right)$ is defined by

$$
\begin{aligned}
\left(\mathbf{w}_{2}, q_{2}\right)= & L^{-1}\left(\mathbf{f} \circ \chi_{\mathbf{w}_{1}+\mathbf{v}_{0}}-\mathbf{f}(0)+\alpha_{1}\left(\mathbf{w}_{1}+\mathbf{v}_{0}, q_{1}+p_{0}\right), \alpha_{3}\left(\mathbf{v}_{0}+\mathbf{w}_{1}\right), 0,0\right) \\
& +L^{-1}\left(-\partial_{t} \underline{\mathbf{v}}_{0}+\nu \Delta \underline{\mathbf{v}}_{0}, \rho_{0}, 0,0\right) .
\end{aligned}
$$

Proposition 3. Let $R_{1}$ be a real number, $R_{1}>0$. There exists a time $T_{1}, 0<T_{1} \leq T_{0}$, such that for all $T \leq T_{1} S_{1}$ has a unique fixed point $(\tilde{v}, \tilde{p})$ in

$$
B_{1}=\left\{(\mathbf{w}, q) \in X_{T, 0}^{r} \mid\left\|(\mathbf{w}, q)-L^{-1}\left(-\partial_{t} \underline{\mathbf{v}}_{0}+\nu \Delta_{\mathbf{v}_{0}}, \rho_{0}, 0,0\right)\right\|_{X_{T}^{r}} \leq R_{1}\right\} .
$$

Indeed, the study of the linear problem (proposition 2) and the estimates of the nonlinear terms give us the estimate $\left\|\left(\mathbf{w}_{2}, q_{2}\right)-L^{-1}\left(-\partial_{t} \underline{\mathbf{v}}_{0}+\nu \underline{\Delta}_{0}, \rho_{0}, 0,0\right)\right\| \leq C T^{\eta}$. We deduce that for a given constant $R_{1}$, there exists a time $T_{1}$ (depending on $\left\|\mathbf{u}_{0}\right\|_{H^{r+1}(\Omega(0))},\left\|\mathbf{v}_{b}\right\|_{H^{r / 2+1}\left(0, T_{0} ; H^{l}(\partial \mathcal{B})\right)}$, f and $R_{1}$ ) such that, for all $T \leq T_{1} \leq T_{0}, S_{1}$ maps $B_{1}$ into itself and is a contraction. This ends the proof of Proposition 3 and the proof of Theorem 2. 
Remark 6. The time $T_{1}$ depends continuously on $\left\|\mathbf{v}_{b}\right\|_{H^{r / 2+1}\left(0, T_{0} ; H^{l}(\partial \mathcal{B})\right)}$ and for all $K>0$, for all $\mathbf{v}_{b}$ such that $\left\|\mathbf{v}_{b}\right\|_{H^{r / 2+1}\left(0, T_{0} ; H^{l}(\partial \mathcal{B})\right)} \leq K$, there exists $T_{K}>0$ such that $T_{1}>T_{K}$.

To summarize, for a strictly positive given constant $R_{1}$, an arbitrary time $T_{0}$, and a velocity $\mathbf{v}_{b}$ given on the rigid body boundary then the problem (12) has a unique smooth solution $(\mathbf{v}, p)$ for small enough time $T \leq T_{1}$. Indeed, let $\left(\mathbf{v}^{\prime}, p^{\prime}\right)$ be another solution, then $\left(\mathbf{v}^{\prime}-\mathbf{v}_{0}, p^{\prime}-p_{0}\right)$ is a fixed point of $S_{1}$; from proposition3 we know that for $T \leq T_{1}$, the fixed point is unique. Now we study the coupled problem.

\section{Coupled problem. Proof of Theorem 1}

Let $\left(\mathbf{w}_{G}, \theta \vec{R}\right) \in H^{r / 2+1}(0, T) \times H^{r / 2+2}(0, T)$, be respectively the center of gravity velocity and the rotation angle of the rigid body. To them we associate the velocity $\mathbf{v}_{b}$ defined by

$$
\mathbf{v}_{b}(x)=\mathbf{w}_{G}+\frac{\mathrm{d}(\exp (\theta[\vec{R} \wedge])}{\mathrm{d} t} \overrightarrow{G_{0} x}, \quad \forall x \in \mathcal{B} .
$$

To the function $\mathbf{v}_{b}$ we associated $(\mathbf{v}, p)=\left(\tilde{\mathbf{v}}+\mathbf{v}_{0}, \tilde{p}+p_{0}\right)$ where $(\tilde{\mathbf{v}}, \tilde{p})$ is the unique fixed point of the mapping $S_{1}$ with $\left.\mathbf{v}\right|_{\partial \mathcal{B}}=\mathbf{v}_{b}$ (proposition 3). For the sake of simplicity we denote by $\vec{\theta}$ the vector $\theta \vec{R}$. Associated to the previous $(\mathbf{v}, p)$, we define now $\left(\overline{\mathbf{w}}_{G}, \overrightarrow{\vec{\theta}}\right)$ solutions of the Newton equations:

$$
\left\{\begin{aligned}
m \frac{\mathrm{d} \overline{\mathbf{w}}_{G}}{\mathrm{~d} t}= & \int_{\partial \mathcal{B}}\left(p-\nu\left(\nabla_{\mathbf{v}}+\left(\nabla_{\mathbf{v}}\right)^{T}\right) \mathbf{v}\right) \operatorname{cof}\left(\nabla \chi_{\mathbf{v}}\right) \cdot \mathbf{n} \\
J \frac{\mathrm{d}^{2} \overrightarrow{\vec{\theta}}}{\mathrm{d} t^{2}}= & -\int_{\partial \mathcal{B}} \rho_{i} \frac{\mathrm{d} \vec{\theta}}{\mathrm{d} t}(t) \wedge\left(\frac{\mathrm{d} \vec{\theta}}{\mathrm{d} t}(t) \wedge\left(\exp ([\vec{\theta} \wedge]) \overrightarrow{G_{0} x}\right)\right) \mathrm{d} x \\
& +\int_{\partial \mathcal{B}}\left(\exp ([\vec{\theta} \wedge]) \overrightarrow{G_{0} x}\right) \wedge\left[\left(p-\nu\left(\nabla_{\mathbf{v}}+\nabla_{\mathbf{v}}{ }^{T}\right) \mathbf{v}\right) \cdot\left(\operatorname{cof}\left(\nabla \chi_{\mathbf{v}}\right) \cdot \mathbf{n}\right)\right] \mathrm{d} x,
\end{aligned}\right.
$$

with the following initial conditions

$$
\overline{\mathbf{w}}_{G}(0)=\mathbf{w}_{0} ; \quad \bar{\theta}(0)=0 ; \quad \frac{\mathrm{d} \vec{\theta}}{\mathrm{d} t}(0)=\vec{\psi}_{0} .
$$

In order to prove the principal result of this paper, we verify that the mapping $S_{2}$

$$
\left(\mathbf{w}_{G}, \vec{\theta}\right) \mapsto\left(\overline{\mathbf{w}}_{G}, \vec{\theta}\right),
$$

has itself also a fixed point. To do so, we first check that the mapping $S_{2}$ is correctly defined, then that, under some assumptions, it maps $B_{2}$ into itself, and is a contraction, with $B_{2}$ defined by:

$$
\begin{aligned}
B_{2}= & \left\{\left(\mathbf{w}_{G}, \vec{\theta}\right) \in H^{r / 2+1}(0, T) \times H^{r / 2+2}(0, T) /\right. \\
& \left.\left\|\mathbf{w}_{G}-\mathbf{w}_{G 0}\right\|_{H^{r / 2+1}(0, T)} \leq R_{2},\left\|\vec{\theta}-\vec{\theta}_{0}\right\|_{H^{r / 2+2}(0, T)} \leq R_{2}\right\} .
\end{aligned}
$$

In the latter definition $R_{2}$ is a given strictly positive constant and $\left(\mathbf{w}_{G 0}, \vec{\theta}_{0}\right)$ are solutions of

$$
\left\{\begin{array}{l}
m \frac{\mathrm{d} \mathbf{w}_{G 0}}{\mathrm{~d} t}=\int_{\partial \mathcal{B}}\left(p_{0}(0)-\nu\left(\nabla+\nabla^{T}\right) \mathbf{u}_{0}\right) \cdot \mathbf{n} \\
J \frac{\mathrm{d}^{2} \vec{\theta}_{0}}{\mathrm{~d} t^{2}}=\int_{\partial \mathcal{B}} \rho \frac{\mathrm{d}}{\mathrm{d} t} \vec{\theta}_{0} \wedge\left(\frac{\mathrm{d}}{\mathrm{d} t} \vec{\theta}_{0} \wedge \overrightarrow{G_{0} x}\right)+\int_{\partial \mathcal{B}} \overrightarrow{G_{0} x} \wedge\left(p_{0}(0)-\nu\left(\nabla+\nabla^{T}\right) \mathbf{u}_{0} \cdot \mathbf{n}\right),
\end{array}\right.
$$


with the following initial conditions

$$
\mathbf{w}_{G 0}(0)=\mathbf{w}_{0} ; \quad \vec{\theta}_{0}(0)=0 ; \quad \frac{\mathrm{d} \vec{\theta}_{0}}{\mathrm{~d} t}(0)=\vec{\psi}_{0}
$$

Let $\left(\mathbf{w}_{G}, \vec{\theta}\right) \in B_{2}$. First, we extend $\left(\mathbf{w}_{G}, \vec{\theta}\right)$ on the time interval $\left(0, T_{0}\right)$, where $T_{0}$ denotes an arbitrary chosen time. To do so, we consider $\mathbf{w}_{G}-\mathbf{w}_{G 0}$. Then, by Lemma 1 , there exists an extension $\phi$ of $\mathbf{w}_{G}-\mathbf{w}_{G 0}$ in $H^{r / 2+1}\left(0, T_{0}\right)$ such that

$$
\|\phi\|_{H^{r / 2+1}\left(0, T_{0}\right)} \leq C\left\|\mathbf{w}_{G}-\mathbf{w}_{G 0}\right\|_{H^{r / 2+1}(0, T)},
$$

where $C$ is a constant independent of $T$. Then $\phi-\mathbf{w}_{G 0}$ is an extension of $\mathbf{w}_{G}$. We denote by $\mathbf{w}_{G}$ this extension. We have

$$
\begin{aligned}
\left\|\mathbf{w}_{G}\right\|_{H^{r / 2+1}\left(0, T_{0}\right)} & \leq\left\|\mathbf{w}_{G 0}\right\|_{H^{r / 2+1}\left(0, T_{0}\right)}+C\left\|\mathbf{w}_{G}-\mathbf{w}_{G 0}\right\|_{H^{r / 2+1}(0, T)} \\
& \leq\left\|\mathbf{w}_{G 0}\right\|_{H^{r / 2+1}\left(0, T_{0}\right)}+C R_{2}
\end{aligned}
$$

Therefore, if $\left\|\mathbf{w}_{G}-\mathbf{w}_{G 0}\right\|_{H^{r / 2+1}(0, T)} \leq R_{2}$, then there exists an extension of $\mathbf{w}_{G}$ to the time interval $\left(0, T_{0}\right)$ such that,

$$
\left\|\mathbf{w}_{G}\right\|_{H^{r / 2+1}\left(0, T_{0}\right)} \leq R_{0},
$$

with the constant $R_{0}$ which depends on $T_{0}, R_{2}, \mathbf{u}_{0}$ and on $\mathbf{f}$, but not on $T$. We use the same argument to extend $\vec{\theta}$ in $H^{r / 2+2}\left(0, T_{0}\right)$. So, if $\left\|\vec{\theta}-\vec{\theta}_{0}\right\|_{H^{r / 2+2}(0, T)} \leq R_{2}$ we obtain an extension of $\vec{\theta}$ to $\left(0, T_{0}\right)$, also denoted by $\vec{\theta}$, such that

$$
\|\vec{\theta}\|_{H^{r / 2+2}\left(0, T_{0}\right)} \leq R_{0}
$$

To these quantities, we associate $\mathbf{v}_{b}$ defined by (52). Since $\vec{\theta} \in H^{r / 2+2}\left(0, T_{0}\right)$, the operator $\exp ([\vec{\theta} \wedge])$ belongs to $H^{r / 2+2}\left(0, T_{0}\right)$. In order to get this result, we write the exponential function with the help of its serie expansion and we note that $H^{r / 2+2}\left(0, T_{0}\right)$ is an algebra. Consequently, $\mathbf{v}_{b} \in H^{r / 2+1}\left(0, T_{0} ; H^{l}(\partial \mathcal{B})\right)$ for any positive real number $l$. Furthermore, we have a bound for $\mathbf{v}_{b}$ :

$$
\begin{aligned}
\left\|\mathbf{v}_{b}\right\|_{H^{r / 2+1}\left(0, T_{0} ; H^{l}(\partial \mathcal{B})\right)} & \leq\left\|\mathbf{w}_{G}\right\|_{H^{r / 2+1}\left(0, T_{0}\right)}+\left\|\frac{\mathrm{d}(\exp ([\vec{\theta} \wedge])}{\mathrm{d} t}\right\|_{H^{r / 2+1}\left(0, T_{0}\right)}, \\
& \leq C\left(R_{0}\right) .
\end{aligned}
$$

To the boundary velocity $\mathbf{v}_{b}$ we associate thanks to the above sections a velocity and a pressure $(\mathbf{v}, p)=$ $\left(\tilde{\mathbf{v}}+\mathbf{v}_{0}^{b}, \tilde{p}+p_{0}\right)$. The velocity and the pressure $(\tilde{\mathbf{v}}, \tilde{p})$ is the fixed point of the mapping ${ }^{1} S_{1}^{b}$. The couple $(\tilde{\mathbf{v}}, \tilde{p})$ is defined over the interval $\left(0, T_{1}^{b}\right)$ with $T_{1}^{b} \leq T_{0}$ and $T_{1}^{b}$ depends on $R_{1}, T_{0},\left\|\mathbf{v}_{b}\right\|_{H^{r / 2+1}\left(0, T_{0} ; H^{l}(\partial \mathcal{B})\right)}\left\|\mathbf{u}_{0}\right\|_{H^{r+1}(\Omega(0))}$ and f. Nevertheless, the time $T_{1}^{b}$ can be the same for all the mappings $S_{1}^{b}$. This results from remark 6 , and from the fact that for $\left(\mathbf{w}_{G}, \vec{\theta}\right) \in B_{2}$, the associated velocity $\mathbf{v}_{b}$ is bounded in $H^{r / 2+1}\left(0, T_{0} ; H^{l}(\partial \mathcal{B})\right)$ with a bound depending only on $R_{0}$. We denote by $T_{1}$ this time. Taking into account the regularities of $\mathbf{v}$ and $p$, there exists a unique solution $\left(\overline{\mathbf{w}}_{G}, \overrightarrow{\vec{\theta}}\right) \in H^{r / 2+1}\left(0, T_{1}\right) \times H^{r / 2+2}\left(0, T_{1}\right)$ of (53). Thus the mapping $S_{2}$ is defined. We shall prove that $S_{2}$ maps $B_{2}$ into itself, for a mass and a moment of inertia sufficiently large. We study $\left\|\overline{\mathbf{w}}_{G}-\mathbf{w}_{G 0}\right\|_{H^{r / 2+1}(0, T)}$ and $\left\|\vec{\theta}-\vec{\theta}_{0}\right\|_{H^{r / 2+2}(0, T)}$. We first restrict the analysis to the $2 D$ case or the case of

\footnotetext{
${ }^{1}$ We add here the exponent $b$ to underline the dependency of $S_{1}^{b}$ and $\mathbf{v}_{0}^{b}$ with respect to $\mathbf{v}_{b}$.
} 
the sphere since then the following equations are satisfied

$$
\left\{\begin{aligned}
m \frac{\mathrm{d}\left(\overline{\mathbf{w}}_{G}-\mathbf{w}_{G 0}\right)}{\mathrm{d} t}= & \int_{\partial \mathcal{B}}\left(p-\nu\left(\nabla_{\mathbf{v}}+\nabla_{\mathbf{v}}^{T}\right) \mathbf{v}\right) \operatorname{cof}\left(\nabla \chi_{\mathbf{v}}\right) \cdot \mathbf{n}-\int_{\partial \mathcal{B}}\left(p_{0}(0)-\nu\left(\nabla+\nabla^{T}\right) \mathbf{u}_{0}\right) \cdot \mathbf{n} \\
J \frac{\mathrm{d}^{2}\left(\vec{\theta}-\vec{\theta}_{0}\right)}{\mathrm{d} t^{2}}= & \int_{\partial \mathcal{B}}\left(\exp ([\vec{\theta} \wedge]) \overrightarrow{G_{0} x}\right) \wedge\left[\left(p-\nu\left(\nabla_{\mathbf{v}}+\nabla_{\mathbf{v}}^{T}\right) \mathbf{v}\right) \operatorname{cof}\left(\nabla \chi_{\mathbf{v}}\right) \cdot \mathbf{n}\right] \\
& -\int_{\partial \mathcal{B}} \overrightarrow{G_{0} x} \wedge\left[p_{0}(0)-\nu\left(\nabla+\nabla^{T}\right) \mathbf{u}_{0} \cdot \mathbf{n}\right]
\end{aligned}\right.
$$

We write $(\mathbf{v}, p)=\left(\tilde{\mathbf{v}}+\mathbf{v}_{0}^{b}, \tilde{p}+p_{0}\right)=\left(\tilde{\mathbf{v}}+\overline{\mathbf{v}}_{0}+\underline{\mathbf{v}}_{0}^{b}, \tilde{p}+p_{0}\right)$. We first have:

$$
\forall T \leq T_{1},\|\tilde{\mathbf{v}}\|_{K_{T}^{r+2}(\Omega(0))}+\|\nabla \tilde{p}\|_{K_{T}^{r}(\Omega(0))} \leq R_{1}+C\left(R_{2}, \mathbf{u}_{0}, \mathbf{f}\right),
$$

since $(\tilde{\mathbf{v}}, \tilde{p}) \in B_{1}$, and since the center of $B_{1}$ is bounded by $C\left(R_{2}, \mathbf{u}_{0}, \mathbf{f}\right)$. Secondly, from (35) and Proposition 2 ,

$$
\left\|\nabla p_{0}\right\|_{K_{T_{0}}^{r}(\Omega(0))}+\left\|\overline{\mathbf{v}}_{0}\right\|_{K_{T_{0}}^{r+2}(\Omega(0))} \leq C\left[\left\|\mathbf{u}_{0}\right\|_{H^{r+1}(\Omega(0))}+\|\mathbf{f}\|_{K_{T_{0}}^{r}(\Omega(0))}+\|\mathbf{z}\|_{H^{r / 2+1}\left(0, T_{0} ; H^{l}(\partial \mathcal{B})\right)}+\left\|\sigma_{0}\right\|_{\hat{K}_{T_{0}}^{r}(\Omega(0))}\right]
$$

We recall that $\sigma_{0}$ belongs to $K_{T_{0}}^{r+2}(\Omega(0))$ and satisfies the initial conditions: $\sigma_{0}(0)=0$ and $\partial_{t} \sigma_{0}(0)=$ $\left(\left(\nabla \mathbf{u}_{0}\right)^{T} \cdot \nabla\right) \mathbf{u}_{0}$. On an other hand, $\mathbf{z}=\left.\mathbf{u}_{0}\right|_{\partial \mathcal{B}}+\alpha(t) \mathbf{w}=\left.\mathbf{u}_{0}\right|_{\partial \mathcal{B}}+\mathbf{w} \int_{\Omega(0)} \sigma_{0}$, thus

$$
\left\|\nabla p_{0}\right\|_{K_{T_{0}}^{r}(\Omega(0))}+\left\|\overline{\mathbf{v}}_{0}\right\|_{K_{T_{0}}^{r+2}(\Omega(0))} \leq C\left(\mathbf{u}_{0}, \mathbf{f}, \mathbf{w}_{0}, \psi_{0}\right) .
$$

Finally, $\underline{\mathbf{v}}_{0}^{b}$ is an extension of $\mathbf{v}_{b}-\mathbf{z}$ which satisfies $\underline{\mathbf{v}}_{0}^{b}(0)=\partial_{t} \underline{\mathbf{v}}_{0}^{b}(0)=0$. Then

$$
\left\|\underline{\mathbf{v}}_{0}^{b}\right\|_{K_{T_{0}}^{r+2}(\Omega(0))} \leq C\left(R_{2}, \mathbf{w}_{0}, \vec{\psi}_{0}, \mathbf{u}_{0}\right) .
$$

This allows to conclude that

$$
\begin{aligned}
m\left\|\overline{\mathbf{w}}_{G}-\mathbf{w}_{G 0}\right\|_{H^{r / 2+1}(0, T)} & \leq C m\left\|\frac{\mathrm{d}}{\mathrm{d} t}\left(\overline{\mathbf{w}}_{G}-\mathbf{w}_{G 0}\right)\right\|_{H^{r / 2}(0, T)} \\
& \leq C\left(R_{1}, R_{2}, \mathbf{u}_{0}, \mathbf{f}, \mathbf{w}_{0}, \vec{\psi}_{0}\right),
\end{aligned}
$$

and

$$
\begin{aligned}
J\left\|\overrightarrow{\bar{\theta}}-\vec{\theta}_{0}\right\|_{H^{r / 2+2}(0, T)} & \leq C J\left\|\frac{\mathrm{d}^{2}}{\mathrm{~d} t^{2}}\left(\overrightarrow{\bar{\theta}}-\vec{\theta}_{0}\right)\right\|_{H^{r / 2}(0, T)} \\
& \leq C\left(R_{1}, R_{2}, \mathbf{u}_{0}, \mathbf{f}, \mathbf{w}_{0}, \vec{\psi}_{0}\right),
\end{aligned}
$$

where the two constants are indeed independant of $m$ and $J$ since they depend only on the right hand sides of (55) that depend only on $\mathbf{v}, p, \mathbf{u}_{0}, p_{0}, \theta$, and not on $m$ and $J$. Consequently, if the body has a mass and a moment of inertia large enough, $S_{2}$ maps $B_{2}$ into itself. In the general $3 D$ case more nonlinearity rises in the equation of $\vec{\theta}$, noticing they appear with a lower order of derivation in time, they can be controlled in small time. We thus have to add in the right hand side of (56) a contribution $C\left(R_{1}, R_{2}, \mathbf{u}_{0}, \mathbf{f}, \mathbf{w}_{0}, \vec{\psi}_{0}\right) \rho\left\|\vec{\theta}_{\theta}-\vec{\theta}_{0}\right\|_{H^{r / 2+1}(0, T)}$ that can be bounded by $C\left(R_{1}, R_{2}, \mathbf{u}_{0}, \mathbf{f}, \mathbf{w}_{0}, \vec{\psi}_{0}\right) \rho T$ so that the same consequence holds at least for a small enough time.

Remark 7. This condition over the characteristics of the rigid body is a sufficient condition to have the stability of $B_{2}$. Nevertheless it does not seem to be very natural. We should have the existence for small time enough of 
a solution for all kind of disc without restrictions. But for the time being we do not have estimates that enable us to eliminate this condition.

We shall show that under similar assumption the mapping $S_{2}$ is a contraction. We consider $\left(\mathbf{w}_{G i}, \vec{\theta}_{i}\right)$ with $i=1,2$ in $B_{2}$ and we associate $\left(\mathbf{v}_{i}, p_{i}\right)=\left(\tilde{\mathbf{v}}_{i}+\mathbf{v}_{0}^{i}, p_{i}+p_{0}\right)$ and $\left(\overline{\mathbf{w}}_{G i}, \overrightarrow{\bar{\theta}}_{i}\right)$. We recall that $\mathbf{v}_{0}^{i}$ is a lifting if the initial and boundary values $\mathbf{v}_{0}$ and $\mathbf{v}_{b i}$ and that this velocity is the sum of : $\underline{\mathbf{v}}_{0}^{i}$ (depending on $\mathbf{v}_{b}^{i}$ ) and $\overline{\mathbf{v}}_{0}$, which is independent of $\mathbf{v}_{b}^{i}$. Therefore again in the case of $2 D$ or of a $3 D$ sphere, if we subtract the equation satisfied by $\left(\mathbf{w}_{G i}, \vec{\theta}_{i}\right)$, for $i=1,2$, and we rearrange the terms so that the differences $\mathbf{v}_{1}-\mathbf{v}_{2}$ and $p_{1}-p_{2}$ appear, we obtain

$$
\begin{aligned}
m \| \overline{\mathbf{w}}_{G 1} & -\overline{\mathbf{w}}_{G 2}\left\|_{H^{r / 2+1}(0, T)}+J\right\| \overrightarrow{\vec{\theta}}_{1}-\overrightarrow{\bar{\theta}}_{2} \|_{H^{r / 2+2}(0, T)} \\
& \leq C\left(\left\|\left(\mathbf{v}_{1}, p_{1}\right)-\left(\mathbf{v}_{2}, p_{2}\right)\right\|_{X_{T}^{r}}+\left\|\exp \left(\left[\vec{\theta}_{1} \wedge\right]\right)-\exp \left(\left[\vec{\theta}_{2} \wedge\right]\right)\right\|_{H^{r / 2+2}(0, T)}\right) \\
& \leq C\left[\left\|\left(\tilde{\mathbf{v}}_{1}, \tilde{p}_{1}\right)-\left(\tilde{\mathbf{v}}_{2}, \tilde{p}_{2}\right)\right\|_{X_{T}^{r}}+\left\|\underline{\mathbf{v}}_{0}^{1}-\underline{\mathbf{v}}_{0}^{2}\right\|_{K_{T_{0}}^{r+2}(\Omega(0))}+\left\|\exp \left(\left[\vec{\theta}_{1} \wedge\right]\right)-\exp \left(\left[\vec{\theta}_{2} \wedge\right]\right)\right\|_{H^{r / 2+2}(0, T)}\right]
\end{aligned}
$$

where $C$ is a constant depending on the data and on $R_{1}$ et $R_{2}$ but which is independent of $T$. Then

$$
\begin{aligned}
& m\left\|\overline{\mathbf{w}}_{G 1}-\overline{\mathbf{w}}_{G 2}\right\|_{H^{r / 2+1}(0, T)}+J\left\|\overrightarrow{\vec{\theta}}_{1}-\overrightarrow{\bar{\theta}}_{2}\right\|_{H^{r / 2+2}(0, T)} \\
& \leq C\left[\left\|\left(\tilde{\mathbf{v}}_{1}, \tilde{p}_{1}\right)-\left(\tilde{\mathbf{v}}_{2}, \tilde{p}_{2}\right)\right\|_{X_{T}^{r}}+\left\|\mathbf{v}_{b 1}-\mathbf{v}_{b 2}\right\|_{\left.K_{T}^{r+3 / 2}(\partial \mathcal{B})\right)}+\left\|\exp \left(\left[\vec{\theta}_{1} \wedge\right]\right)-\exp \left(\left[\vec{\theta}_{2} \wedge\right]\right)\right\|_{H^{r / 2+2}(0, T)}\right] .
\end{aligned}
$$

Now we estimate $\left\|\left(\tilde{\mathbf{v}}_{1}, \tilde{p}_{1}\right)-\left(\tilde{\mathbf{v}}_{2}, \tilde{p}_{2}\right)\right\|_{X_{T}^{r}}$ with respect to $\mathbf{v}_{b 1}-\mathbf{v}_{b 2}$, with

$$
\mathbf{v}_{b i}=\mathbf{w}_{G i}+\frac{\mathrm{d}\left(\exp \left(\left[\vec{\theta}_{i} \wedge\right]\right)\right)}{\mathrm{d} t} \overrightarrow{G_{0} x}, i=1,2 .
$$

We recall that

$$
\begin{cases}\partial_{t} \tilde{\mathbf{v}}_{i}-\nu \Delta \tilde{\mathbf{v}}_{i}+\nabla \tilde{p}_{i}=\mathbf{f} \circ \chi_{\tilde{\mathbf{v}}_{i}+\mathbf{v}_{0}^{i}-\mathbf{f}(0)+\alpha_{1}\left(\mathbf{v}_{0}^{i}+\tilde{\mathbf{v}}_{i}, p_{0}+\tilde{p}_{i}\right)} \quad-\partial_{t} \underline{\mathbf{v}}_{0}^{i}+\nu \underline{\mathbf{v}}_{0}^{i} & \text { in } \Omega(0) \\ \operatorname{div} \tilde{\mathbf{v}}_{i}=\alpha_{2}\left(\mathbf{v}_{0}^{i}+\tilde{\mathbf{v}}_{i}\right)-\operatorname{div} \mathbf{v}_{0}^{i} & \text { in } \Omega(0) \\ \tilde{\mathbf{v}}_{i}=0 & \text { on } \Gamma_{0} \cup \partial \mathcal{B} \\ \tilde{\mathbf{v}}_{i}(0)=0 & \text { in } \Omega(0),\end{cases}
$$

We subtract the equations satisfied by $\left(\tilde{\mathbf{v}}_{1}, \tilde{p}_{1}\right)$ and $\left(\tilde{\mathbf{v}}_{2}, \tilde{p}_{2}\right)$. The approach we use at the previous section and lemma 7, 8 and 9 leads to the following estimate:

$$
\begin{aligned}
\left\|\left(\tilde{\mathbf{v}}_{1}, \tilde{p}_{1}\right)-\left(\tilde{\mathbf{v}}_{2}, \tilde{p}_{2}\right)\right\|_{X_{T}^{r}} \leq & C T^{\eta}\left\|\left(\tilde{\mathbf{v}}_{1}, \tilde{p}_{1}\right)-\left(\tilde{\mathbf{v}}_{2}, \tilde{p}_{2}\right)\right\|_{X_{T}^{r}}+C\left\|\underline{\mathbf{v}}_{0}^{1}-\underline{\mathbf{v}}_{0}^{2}\right\|_{K_{T}^{r+2}(\Omega(0))} \\
& +C\left\|\operatorname{div}\left(\underline{\mathbf{v}}_{0}^{1}-\underline{\mathbf{v}}_{0}^{2}\right)\right\|_{\hat{K}_{T}^{r}(\Omega(0))}
\end{aligned}
$$

Using the definition of $\underline{\mathbf{v}}_{0}^{i}$ for $i=1,2$, we get

$$
\left\|\left(\tilde{\mathbf{v}}_{1}, \tilde{p}_{1}\right)-\left(\tilde{\mathbf{v}}_{2}, \tilde{p}_{2}\right)\right\|_{X_{T}^{r}} \leq C T^{\eta}\left\|\left(\tilde{\mathbf{v}}_{1}, \tilde{p}_{1}\right)-\left(\tilde{\mathbf{v}}_{2}, \tilde{p}_{2}\right)\right\|_{X_{T}^{r}}+C\left\|\mathbf{v}_{b 1}-\mathbf{v}_{b 2}\right\|_{H^{r / 2+1}\left(0, T ; H^{l}(\partial \mathcal{B})\right)}
$$

For all $T \leq T_{1}$, we have $C T^{\eta}<1$ thanks to the way we choose $T_{1}$. Then there exists a constant we denote by $C$ such that:

$$
\left\|\left(\tilde{\mathbf{v}}_{1}, \tilde{p}_{1}\right)-\left(\tilde{\mathbf{v}}_{2}, \tilde{p}_{2}\right)\right\|_{X_{T}^{r}} \leq C\left\|\mathbf{v}_{b 1}-\mathbf{v}_{b 2}\right\|_{H^{r / 2+1}\left(0, T ; H^{l}(\partial \mathcal{B})\right)}
$$


The estimates (57) and (59) yield

$$
\begin{aligned}
& m\left\|\overline{\mathbf{w}}_{G 1}-\overline{\mathbf{w}}_{G 2}\right\|_{H^{r / 2+1}(0, T)}+J\left\|\overrightarrow{\vec{\theta}}_{1}-\overrightarrow{\vec{\theta}}_{2}\right\|_{H^{r / 2+2}(0, T)} \leq \\
& C\left\|\mathbf{v}_{b 1}-\mathbf{v}_{b 2}\right\|_{H^{r / 2+1}\left(0, T ; H^{l}(\partial \mathcal{B})\right)}+\left\|\exp \left(\left[\vec{\theta}_{1} \wedge\right]\right)-\exp \left(\left[\vec{\theta}_{2} \wedge\right]\right)\right\|_{H^{r / 2+2}(0, T)}
\end{aligned}
$$

Furthermore,

$$
\left\|\mathbf{v}_{b 1}-\mathbf{v}_{b 2}\right\|_{H^{r / 2+1}\left(0, T ; H^{l}(\partial \mathcal{B})\right)} \leq\left\|\mathbf{w}_{G 1}-\mathbf{w}_{G 2}\right\|_{H^{r / 2+1}(0, T)}+\left\|\exp \left(\left[\vec{\theta}_{1} \wedge\right]\right)-\exp \left(\left[\vec{\theta}_{2} \wedge\right]\right)\right\|_{H^{r / 2+2}(0, T)} .
$$

We shall estimate $\left\|\exp \left(\left[\vec{\theta}_{1} \wedge\right]\right)-\exp \left(\left[\vec{\theta}_{2} \wedge\right]\right)\right\|_{H^{r / 2+2}(0, T)}$ with respect to $\left\|\vec{\theta}_{1}-\vec{\theta}_{2}\right\|_{H^{r / 2+2}(0, T)}$. We want to apply Lemma 5 ii). We have

$$
\left\|\exp \left(\left[\vec{\theta}_{1} \wedge\right]\right)-\exp \left(\left[\vec{\theta}_{2} \wedge\right]\right)\right\|_{H^{r / 2+2}(0, T)} \leq C\left(T_{0}\right) \| \frac{\mathrm{d}^{2}}{\mathrm{~d} t^{2}}\left(\exp \left(\left[\vec{\theta}_{1} \wedge\right]\right)-\exp \left(\left[\vec{\theta}_{2} \wedge\right]\right) \|_{H^{r / 2}(0, T)}\right.
$$

We recall that $\exp \left(\left[\vec{\theta}_{i} \wedge\right]\right)$ are matrices depending on the cosines and the sinus of the various components of the vector $\theta_{i} \vec{R}_{i}$. So using the series expansion of cosines and sinus, combining the terms in order to make appearing the difference $\vec{\theta}_{1}-\vec{\theta}_{2}$ and then applying lemma 5 ii), it comes

$$
\left\|\frac{\mathrm{d}^{2}}{\mathrm{~d} t^{2}}\left(\exp \left(\left[\vec{\theta}_{1} \wedge\right]\right)-\exp \left(\left[\vec{\theta}_{2} \wedge\right]\right)\right)\right\|_{H^{r / 2}(0, T)} \leq C\left(R_{2}\right)\left\|\vec{\theta}_{1}-\vec{\theta}_{2}\right\|_{H^{r / 2+2}(0, T)} .
$$

Thus

$$
\left\|\mathbf{v}_{b 1}-\mathbf{v}_{b 2}\right\|_{H^{r / 2+1}\left(0, T ; H^{l}(\partial \mathcal{B})\right)} \leq\left\|\mathbf{w}_{G 1}-\mathbf{w}_{G 2}\right\|_{H^{r / 2+1}(0, T)}+C\left(R_{2}\right)\left\|\vec{\theta}_{1}-\vec{\theta}_{2}\right\|_{H^{r / 2+2}(0, T)} .
$$

The estimates (60) and (61) lead to

$$
\begin{gathered}
m\left\|\mathbf{w}_{G 1}-\mathbf{w}_{G 2}\right\|_{H^{r / 2+1}(0, T)}+J\left\|\theta_{1}-\theta_{2}\right\|_{H^{r / 2+2}(0, T)} \leq \\
C\left(\left\|\mathbf{w}_{G 1}-\mathbf{w}_{G 2}\right\|_{H^{r / 2+1}(0, T)}+C\left(R_{2}\right)\left\|\theta_{1}-\theta_{2}\right\|_{H^{r / 2+2}(0, T)}\right) .
\end{gathered}
$$

Then the mapping $S_{2}$ is a strict contraction for a mass and a moment of inertia of the disc large enough. The general $3 D$ is treated as before. This conclude the proof of Theorem 1.

Acknowledgements. This work has been benefited of the scientific frame of the GDR $\mathrm{n}^{\circ} 1135$ and the PICS 478, in particular we want to thank P.G. Ciarlet*, H. Le Dret, M. Esteban, C. Farhat, R. Ohayon for enlighting discussions.

\section{REFERENCES}

[1] R.A. Adams, Sobolev spaces. Academic Press, New York NY, San Francisco CA, London (1975).

[2] G. Allain, Un problème de Navier-Stokes avec surface libre. Thèse de troisième cycle de l'Université Paris VI, France (1983).

[3] G. Allain, Small-time existence for the Navier-Stokes equations with a free surface. Appl. Math. Optim., 16 (1987) 37-50.

[4] J.T. Beale, The Initial Value Problem for the Navier-Stokes Equation with a Free Surface. Comm. Pure Appl. Math., XXXIV (1981) 359-392.

[5] H. Brezis, Analyse fonctionnelle: Théorie et applications. Masson, Paris (1983).

[6] C. Conca, J. San Martin and M. Tucsnak, Motion of a rigid body in viscous fluid. C. R. Acad. Sci. Paris Série I 32 (1999) $473-478$.

\footnotetext{
${ }^{*}$ These results were announced in the note of C. R. Acad. Sci. Paris [12].
} 
[7] B. Desjardins and M.J. Esteban, Existence of weak solutions for the motion of rigid bodies in a viscous fluid. Arch. Ration. Mech. Anal.146 (1999) 59-71.

[8] B. Desjardins and M.J. Esteban, On weak solutions for fluid-rigid structure interaction: compressible and incompressible models. Comm. Partial Differ. Eq. (to appear).

[9] G. Duvaut, Mécanique des milieux continus. Masson, Paris, Milan, Barcelone, Mexico (1990).

[10] V. Girault and P.A. Raviart, Finite Element Methods for Navier-Stokes Equations. Springer-Verlag, Berlin Heidelberg (1986).

[11] R. Glowinski and B. Maury, Fluid-particule flow: a symmetric formulation. C. R. Acad. Sci. Paris Sér. I Math. t. 324, (1997) 1079-1084.

[12] C. Grandmont and Y. Maday, Existence de solutions d'un problème de couplage fluide-structure bidimensionnel instationnaire. C. R. Acad. Sci. Paris Sér. I Math. t. 326, (1998) 525-530.

[13] J. Heywood and R. Rannacher, Finite-element approximation of the nonstationnary Navier-Stokes problem. Part III. Smoothing property and higher order error estimates for spatial discretisation. SIAM J. Numer. Anal. 25 (1988) 489-512.

[14] J.L. Lions and E. Magenes, Problèmes aux limites non homogènes et applications. T. I et II, Dunod, Paris (1968).

[15] D. Serre, Chute libre d'un solide dans un fluide visqueux incompressible : Existence. Japan J. Appl. Math. 4 (1987) 33-73.

[16] V.A. Solonnikov, Solvability of a Problem on the Motion of a Viscous Incompressible Fluid Bounded by a Free Surface. Math. USSR Izvestiya 4-1 (1977) 1388-1424.

[17] V.A. Solonnikov, On the Transiant Motion of an Isolated Volume of Viscous Incompressible Fluid. Math. USSR Izvestiya 31 (1988) 381-405.

[18] V.A. Solonnikov, Unsteady motion of a finite mass of fluid, bounded by a free surface. J. Soviet Math. 40 (1988) 672-686.

[19] R. Temam, Navier-Stokes Equations. North-Holland Publishing Company (1977). 